\title{
Spatial averaging of velocity measurements in wall-bounded turbulence: single hot-wires
}

\author{
Jimmy Philip, Nicholas Hutchins, Jason P Monty and \\ Ivan Marusic \\ Department of Mechanical Engineering, The University of Melbourne, Parkville, VIC \\ - 3010, Australia. \\ E-mail: jimmyp@unimelb.edu.au or jimmy.philip@gmail.com
}

\begin{abstract}
Recent advancements in velocity measurements to understand high Reynolds number $(R e)$ wall-turbulence have pushed the boundaries of sensor size required to resolve the smallest scales. We present here a framework for studying the effect of finite sensor size on velocity measurements, and scrutinise in detail the behaviour of single-wire hot-wires. Starting with a general linear filter, expressions for the filtered correlation, spectrum and the corresponding variance are derived. Considering the special case of a box-type filter and a simple model for the twopoint correlation, theoretical results are developed, which compare favourably with the numerical simulation of hot-wires based on the turbulent channel flow Direct Numerical Simulation databases. The results clarify the reason why previous studies found the approximate shape of the spectra not resolved by hot-wires as Gaussian. The length scale based on the correlation over the sensor length is found to be the appropriate length scale for characterising averaging due to finite sensor size. The efficacy of the linear box-filter is established by comparing the numerical simulation of hot-wires with experiments conducted at matched sensor lengths and $R e$ in a channel flow, at least for hot-wire lengths less than 40 in viscous scaling. Finally, a model of the streamwise two-point correlation is presented, which is employed to estimate the filtering effect on the peak of the streamwise velocity variances for a range of $R e$, and the model results compare favourably with that obtained from measurements. Even though the theoretical results are compared here in the case of wall-turbulence, they are suitable for hot-wire measurements in turbulent flows in general.
\end{abstract}

\section{Introduction}

Eulerian velocity is probably the single-most important field measured to improve our understanding of any fluid-mechanical system, and the study of turbulence is no different. From the second quarter of the twentieth century, hot-wire anemometry (HWA) has remained the main work-horse for Eulerian velocity measurements in advancing our knowledge of turbulence. Its ubiquitous use in the study of turbulence is no surprise owing to its high temporal response, relatively low cost, long-time measurement capability, and most importantly the wealth of information that has been gathered over years, making it one of the most reliable instruments for velocity 
measurements [Perry, 1982, Bruun, 1995]. Over the past few decades, with the advent of high speed computers and fast imaging sensors, the technique of Particle Image Velocimetry (PIV) and its variants are employed at an increasing pace within the turbulence community and outside due to the ease with which multi-component velocity measurements can be made instantaneously over a substantial region of space [e.g. Adrian and Westerweel, 2011]. Both the techniques are complementary; PIV provides the spatial information that is important for the structural/organized-motion aspects of turbulence whereas hot-wire anemometry is presently irreplaceable for the statistical description of turbulence wherein moments of all orders are required to provide a complete description of turbulence.

The inherent need for a 'point' velocity measurement is quite clear when using either of the techniques. However, the hot-wire length over which heat is transferred is finite and so is the area of the interrogation window in PIV over which the correlation is calculated. The finite sensor length must be considered against the smallest scales in the turbulent flow that need to be resolved. This could be the Kolmogorov length scale $(\eta)$ or the viscous length scale $\left(\nu / u_{\tau}\right.$, where $\nu$ is kinematic viscosity of the fluid and $u_{\tau}$, the friction velocity) in wall-bounded flows, both of which decrease with increasing Reynolds number $(R e)$. One way to approach the 'point' velocity measurement is to make the sensors smaller, which is eventually limited due to the unavailability of smaller wires and handling issues in HWA, and the compromise that has to be made on the overall region of velocity measurement in PIV. The nano-scale HWA by Bailey et al. [2010] has pushed the conventional hot-wire limits. Even so the sensor length can still be many times larger than the smallest scales of turbulence for the highest $R e$ that can be obtained in the laboratory. Another alternative for point measurement is to increase the length scales of the turbulence, while maintaining high $R e$; this calls for very large windtunnels such as the High Reynolds Number Boundary Layer Wind Tunnel (HRNBLWT) at the University of Melbourne [e.g., Nickels et al., 2007] and the wind-tunnel at Ecole Centrale de Lille [e.g., Carlier and Stanislas, 2005]. Even with such small hot-wires and/or large facilities, the problem of spatial resolution of sensors is unavoidable [see for example Hutchins et al., 2009].

Spatial attenuation due to the finiteness of the hot-wire was recognized early on, from Dryden et al. [1937], followed by Frenkiel [1949], Corrsin and Kovasznay [1949], Uberoi and Kovasznay [1953], Frenkiel [1954] and Wyngaard [1968], wherein the relationship between the filtered quantities (in particular, the variance of the streamwise velocity, $\tilde{u}_{r m s}^{2}$ and spectrum, $\tilde{\phi}_{11} \ddagger$ ) and the unfiltered quantities are presented. (Note that spatially averaged/filtered quantities are presented with a tilde throughout.) The relationships are derived following different paths though leading to the same expression, however, always concentrating on the homogeneous isotropic turbulence (HIT) and mostly for single hot-wires. For a more recent work concerning the effect of the anisotropy in the spectra on single hot-wires, see Cameron et al. [2010].

$\ddagger$ Note that even though $\tilde{\phi}_{11}$ is the 'power spectrum' of $\tilde{u}_{1}$ or the Fourier transform of the two-point correlation, we refer to it simply the 'spectrum' following the usual practice in wall-turbulence. 
For wall-bounded turbulent shear flows, the spatial resolution issues could be quantified reliably only after the availability of Direct Numerical Simulation (DNS) databases. Moin and Spalart [1987] marks the first study of its kind at $R e_{\tau}$ of 180 (where, $R e_{\tau}=u_{\tau} \delta / \nu$, and $\delta$ is the turbulent boundary layer thickness). A more detailed investigation was undertaken by Suzuki and Kasagi [1992] at $R e_{\tau}=150$ for both single and cross-wire (however, concentrating primarily on the effect of separation rather than spatial averaging for cross-wires). More recently Chin et al. [2009] and Chin et al. [2011] used the database of del Álamo et al. [2003] at a $R e_{\tau}$ of 934, wherein the filtering effect of a single hot-wire is studied. A correction methodology for $u_{r m s}^{2}$ and $\phi_{11}$ are provided with a third order polynomial and a Gaussian fit, respectively, given the filtered quantities, $\tilde{u}_{r m s}^{2}$ and $\tilde{\phi}_{11}$. A correction scheme for $u_{r m s}^{2}$ has also been proposed by Monkewitz et al. [2010] and Smits et al. [2011]. Recently Segalini et al. [2011a] considered a single hot-wire and derived relations for $\tilde{u}_{r m s}^{2}$ in terms of the Taylor micro-scale, studying the effect of averaging using the DNS database at $R e_{\tau}=550$ in a channel flow. A more comprehensive review of literature to 2007 is presented by ComteBellot and Foss [2007] regarding hot-wire averaging issues. It should be mentioned that experimentally, hot-wire spatial resolution issues for single normal wires have been investigated extensively [e.g., Johansson and Alfredsson, 1983, Ligrani and Bradshaw, 1987, Hutchins et al., 2009, Ng et al., 2011]. While the experimental studies provide the attenuation in variance and spectrum due to finite hot-wire length, pin-pointing the sources of errors are considerably more difficult. Their introductions also furnish other issues related to single hot-wires and a more complete reference for experimental investigations. However, there are no such detailed experimental investigations available for guidance in cross-wires. For PIV, Saikrishnan et al. [2006] is probably the first and the only investigation employing a DNS database to study the spatial averaging issues for a dual plane PIV. On the experimental side, Lavoie et al. [2007] have developed an expression for $\tilde{u}_{r m s}^{2}$ for planar PIV and used it to correct their measurements in HIT.

\subsection{A framework for the study for spatial averaging of sensors}

For the purpose of simplifying the investigation of spatial averaging, it is convenient to concentrate on special regions of the wall-bounded flows separately. Figure 1 shows one of the walls of the channel flow, or the flat plate of a zero-pressure-gradientturbulent-boundary-layer (ZPG-TBL) with the mean velocity only a function of the wallnormal distance, $U(z)$, and homogeneous in the $x y$-plane. $\S$ Throughout the paper, $x, y$ and $z$ represent the streamwise, spanwise and wallnormal directions, and $u, v$ and $w$, the corresponding velocities. Length and velocity scales are normalised by $\nu / u_{\tau}$ and $u_{\tau}$ respectively, and represented by plus, ${ }^{+}$symbols, e.g., $l^{+}=l u_{\tau} / \nu$ and $u^{+}=u / u_{\tau}$.

$\S$ Note that in a ZPG-TBL the boundary layer is continuously 'developing', therefore, the $x y$-plane is not homogeneous, however, for our purpose it is sufficient that the variation of the mean flow in $x$ be much smaller than that in $z$ over a region of approximately the sensor size. This is true for ZPG-TBL. Therefore, in the present context, any further reference to the difference between a channel flow and ZPG-TBL is unnecessary. 


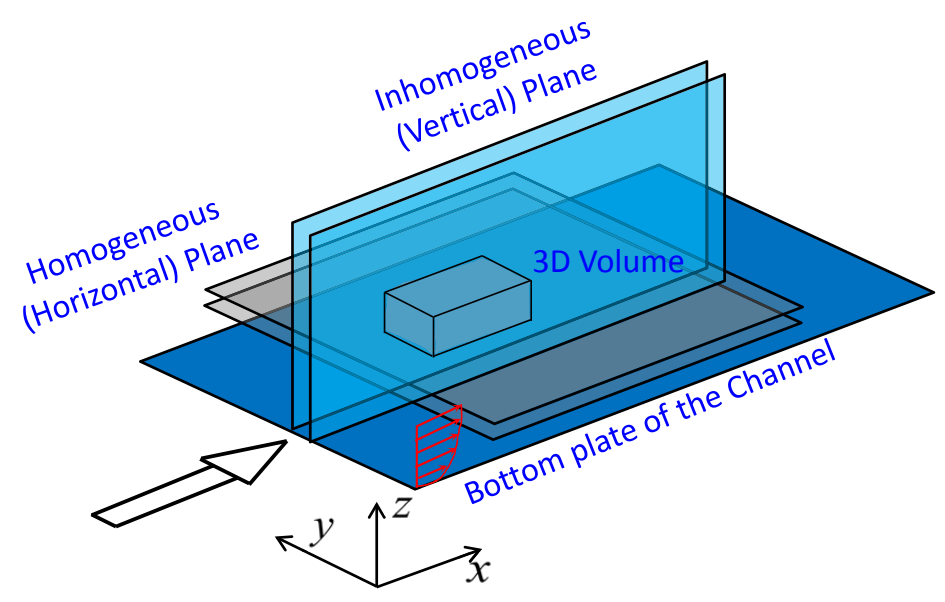

Figure 1. A schematic for the classification of different regions in a wall-bounded turbulent flow which simplifies the study of averaging effect. Multiple horizontal and vertical planes indicate locations of cross-wires, where the two wires are generally situated on two different planes, except the $\vee$-probe (c.f., figure 2c) which is on a single plane.

The study of sensors can be separated into three different categories, depending on the variation of the mean velocity encountered by the sensors namely, the sensors on the homogeneous plane/s (or the horizontal $x y$-plane), those on the inhomogeneous plane/s (or the vertical $x z$-plane) and the sensors which occupy an entire volume. The different regions are shown schematically in figure 1.

Note that for the sensors on a single homogeneous plane there is no mean flow variation, consequently there is no effect of wallnormal shear and the correlation and the spectrum are well defined. This is the simplest case. A single hot-wire, a PIV plane (with a very small depth of focus or laser sheet thickness) and a $\vee$-probe belong to this category. These are shown as schematics in Figure 2(a), (b) and (c) respectively. Further complexity can be added if the sensor is located on two (or multiple) homogeneous $(x y)$ planes. In this case even though the mean flow between the two planes are different, there is no mean flow variation over the sensor itself. The $\times$-probe for $u-v$ (streamwisespanwise) velocity measurements belongs here, and is shown schematically in figure $2(d)$.

The next category involves planes with mean shear, i.e. the $x z$-plane/s. Figure 3(a), (b) and (c) present a single hot-wire, a PIV plane and an $\times$-probe for the measurement of $u-w$ (streamwise-wallnormal) velocities, respectively, which reside on vertical plane/s. Note that the configuration of single hot-wire shown here is not usual, however, in cases where there is an inflow from the wall, the use of this type is not uncommon; moreover, this simple design is a good starting point to understand the effect of mean velocity shear without complications of multiple wires. Again, the PIV plane is assumed to be of almost zero thickness. The third category of volume averaging involves a more realistic PIV measurement plane where the thickness of the laser sheet, or the depthof-focus is finite. Other 3D measurement techniques such as tomographic PIV can also 


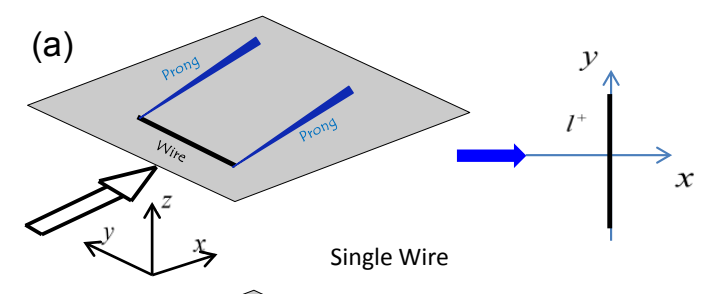

(b)
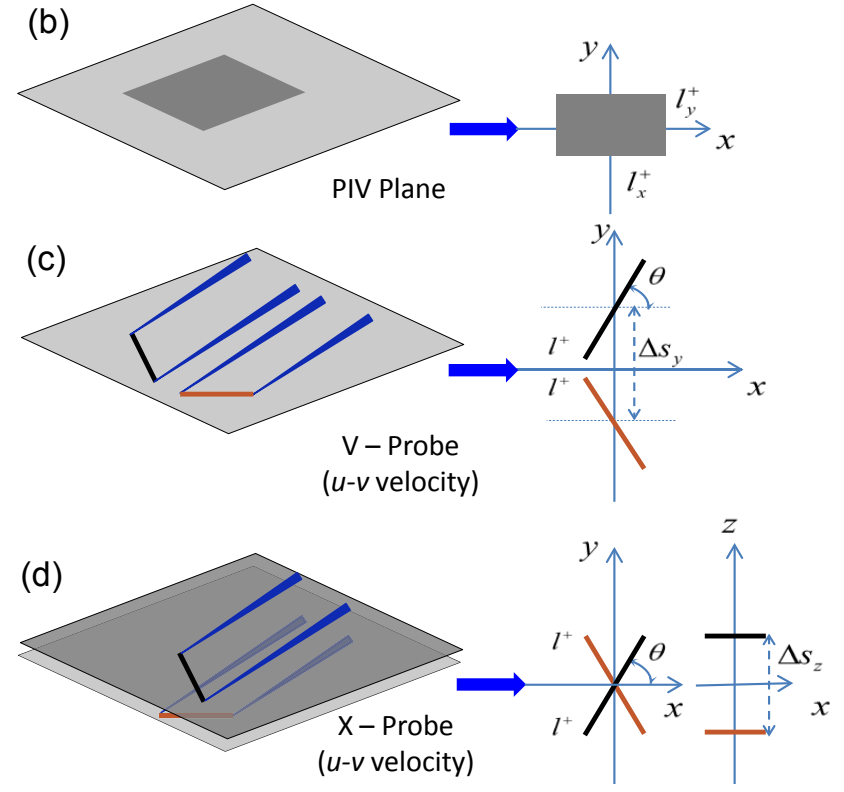

Figure 2. Sensors in the homogenous plane/s (i.e., fixed $z$-location/s). (a) A single hot-wire with the wire placed in the spanwise direction. (b) A PIV interrogation region in the horizontal plane. (c) A $\vee$-probe for the measurement of $u$ - $v$ (streamwisespanwise) velocity. (d) An $\times$-probe, again, for $u-v$ velocity measurements. Note that, in the schematics, the prongs are depicted as inclined to the horizontal plane, in accordance with the usual experimental configurations.

be assumed to belong to this category.

\subsection{Objectives}

Consider the schematic of a single hot-wire in figure 4, which is a hypothetical case wherein the instantaneous velocity field, $u$, is uniform across the hot-wire, resulting in the velocity correlation of unity over the entire length of the wire. This is the case where there is no spatial averaging because the instantaneous velocity at any point on the wire is perfectly correlated with any other point. Figure 4(b) is the realistic case where the velocity is non-uniform over the length, correlation drops and consequently the effects of spatial averaging are present. Therefore, the knowledge of the correlation provides a clue to the averaging of the velocity. The first objective of the present work is to relate the variance, $\tilde{u}_{r m s}^{2}$, the two-point correlation and spectrum of the averaged velocity field to the un-averaged quantities for the case of single hot-wires. These relations can also be extended for the case of $\vee$ and $\times$ wires without excessive difficulties [see, e.g. Philip et al., 2013]. Spatial filtering in hot-wires implies 'line' averaging of the velocity field. An extension to two-dimensional planar averaging, relevant for PIV and a three- 


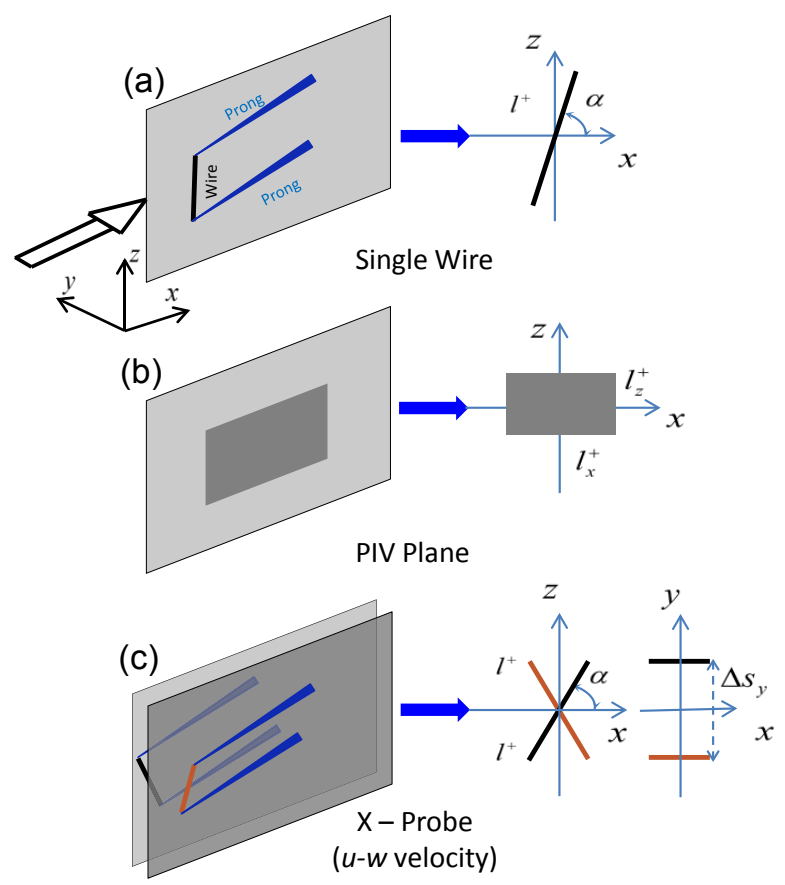

Figure 3. Sensors in the vertical plane (i.e., fixed $y$-location). (a) A single hot-wire with wire place in the vertical plane (not necessarily along $z$ ). (b) A PIV interrogation region in the vertical plane. (c) An $\times$-probe, for $u w$ (streamwise-wallnormal) velocity.

(a)

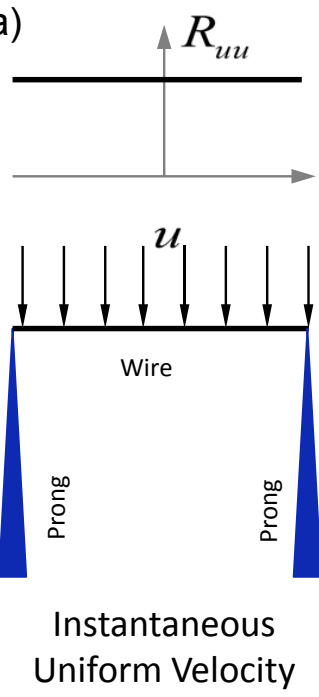

(b)
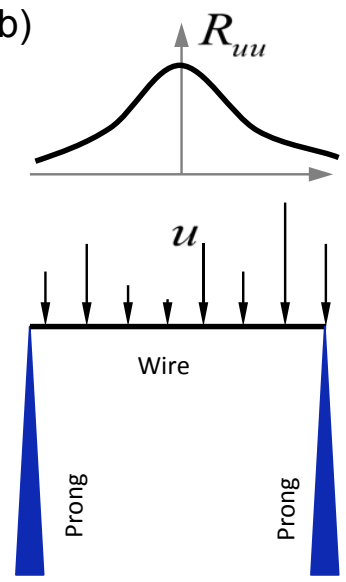

Instantaneous non-Uniform Velocity

Figure 4. Schematic of the spatial averaging process in a single hot-wire. (a) An ideal motion of fluid wherein the instantaneous velocity is constant, correlation is a constant equal to one, and consequently no averaging effect. (b) A realistic field where the correlation is not equal to one over the hot-wire and in turn has a filtering effect. 
dimensional case applicable for volume measurement techniques, such as tomographic PIV is also foreseeable. Since distribution of correlations plays a major role in spatial averaging, the second objective is to find the various correlations (from the channel flow DNS of del Álamo et al. [2003] at $R e_{\tau}$ of 180, 550, 934 and 2003) that are relevant to the particular averaging problem. From this we develop simple models of correlations to understand the effect of averaging analytically for single hot-wires. The final objective is to assess the efficacy of the linear box-filtering technique applied to the DNS database in light of actual experimental evidence.

\subsection{Organisation of the paper and some detail of the DNS database}

The present paper concentrates on the averaging issue related to single wires, later extendable to other wire configurations where the sensor is located in the homogeneous plane (wherein the mean velocity is constant) and in the inhomogeneous plane. Section 2 includes the bulk of the work. This section starts with some analytical considerations relevant for single-wire averaging, followed by showing the effect of filtering on correlation and spectrum. A simple model for correlation is built over a small region (of the hot-wire), from which we can analytically predict the filtered quantities. Initially a single homogeneous plane, at $z^{+}=15$ at $R e_{\tau}=934$ is studied in detail, subsequently the effect of other wall-normal locations and the Reynolds number effect are also studied. Section 2 ends with a comparison of the DNS filtering with actual experimental results, some of which are taken from $\mathrm{Ng}$ et al. [2011] and some newly conducted. Finally, section 3 presents a summary of the work and further conclusions.

DNS databases are ideal for studying spatial averaging issues, and in the current study we employ the database of del Álamo et al. [2003] at $R e_{\tau}=934$, which has a spatial discretization of Fourier $\times$ Fourier $\times$ Chebychev with grid points $N_{x} \times N_{y} \times N_{z}=$ $3072 \times 2304 \times 385$ in streamwise, spanwise and wallnormal directions. The computational domain is $8 \pi h$ units in the streamwise direction and $3 \pi h$ units in the spanwise direction, where $h$ is the half channel height. After de-aliasing in the Fourier domain, the equivalent resolution in the real domain for streamwise and spanwise directions is $\Delta x^{+} \times \Delta y^{+} \approx$ $11.46 \times 5.73$. However, due to the $3 / 2$ de-aliasing rule, in the real domain the available database has a velocity field on an interpolated grid with the resolution $\Delta x^{+} \times \Delta y^{+} \approx$ $7.6 \times 3.8$, which is the resolution that is presented in figures below, to be consistent with previous studies, e.g., Chin et al. [2009]. In the wall-normal direction, the grid spacing increases from $\Delta z^{+} \approx 0.03$ at the wall to a maximum 7.6 at the centre of the channel.

\section{Single hot-wire in the homogeneous plane}

The present analysis concentrates on the single hot-wire due to its importance, and also because the understanding gained and the analysis performed here can easily be carried across to other sensors. 


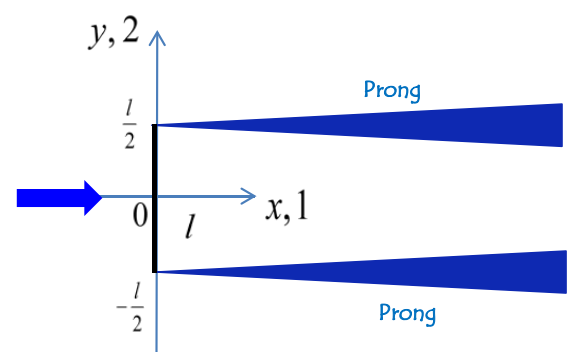

Figure 5. Schematic of a single hot-wire in the $x y$-plane.

\subsection{Some analytical considerations}

Consider figure 5, which shows the schematic of a single hot-wire in the $x y$-plane placed perpendicular to the mean streamwise velocity with a length $l$. The origin is at the center of the wire with a half length of wire, $l / 2$ in both positive and negative $y$-directions. Depending on the convention, directions $x$ and $y$ are also sometimes indicated by 1 and 2 , respectively. A 'simple' averaging of the instantaneous velocity, $u$ along the length of the wire results in,

$$
\tilde{u}=\frac{1}{l} \int_{-l / 2}^{l / 2} u(y+s) \mathrm{d} s,
$$

where, $s$ is along the homogeneous $y$-direction. This is a 'box' filter, and is the one used in previous investigations employing DNS databases. However, there are occasions where one has to resort to a different filter; for example, when the temperature profile changes drastically along the wire due to mean shear, or, due to the variations of light intensity in PIV. Therefore, considering an arbitrary filter, $b(s)$, normalized such that the area is $l$, the averaged velocity field becomes a convolution of the un-averaged velocity field, $u$, and $b$,

$$
\tilde{u}=\frac{1}{l} \int u(y-s) b(s) \mathrm{d} s,
$$

where the integral extends over the domain of the integrands which is typically from $-\infty$ to $\infty$, or, in the symbolic form,

$$
\tilde{u}=\frac{1}{l} u * b
$$

where, $*$ is the convolution integral [e.g., Bracewell, 2000], and its definition is evident by comparing eqns (2) and (3). Decomposing the instantaneous velocity $u$ into mean $(U)$ and fluctuations $(u)$, as $u=U+u^{\prime}$, results in equations for spatially-averaged or filtered mean and fluctuation fields as,

$$
\tilde{U}=\frac{1}{l} U * b,
$$

and,

$$
\tilde{u^{\prime}}=\frac{1}{l} u^{\prime} * b
$$


respectively. An important conclusion can immediately be deduced from the above two equations. The averaged mean velocity from a linear filter is affected only by the unfiltered mean, and likewise the filtered fluctuating component depends only on the unfiltered fluctuations and not on the unfiltered mean. This produces non-intuitive results. For example when considering $\times$-wires (c.f., figure 2) with wires on two different $x y$-planes, where it might seem that the variances may somehow be affected by the fact that the two wires experience different mean velocities. However, this is not the case according to eqn (5), which indicates that only fluctuating components dictate filtered fluctuations and not the mean quantities, at least in the context of linear-filtering.

Once the filtered fluctuating field $\tilde{u}^{\prime}$ is obtained, the (non-normalized) two-point correlation between two averaged velocity fields can be defined as,

$$
\begin{aligned}
\tilde{R}_{i j}(r) & =\lim _{L \rightarrow \infty} \frac{1}{L} \int{\tilde{u^{\prime}}}_{i}(s){\tilde{u^{\prime}}}_{j}(r+s) \mathrm{d} s \\
& =\left\langle{\tilde{u^{\prime}}}_{i}(s) \tilde{u^{\prime}}{ }_{j}(r+s)\right\rangle
\end{aligned}
$$

where, the integral is over the length of the field, $L$, the subscripts $i$ and $j$ vary from 1 to 3 corresponding to the velocities $u, v$ and $w$, and the angled brackets, \langle\rangle denote ensemble averages. This can be rewritten in a more compact form with the 'usual' definition of correlation [e.g., Bracewell, 2000] $\star$, as,

$$
\tilde{R}_{i j}(r)=\lim _{L \rightarrow \infty} \frac{1}{L}\left({\tilde{u^{\prime}}}_{i} \star{\tilde{u^{\prime}}}_{j}\right) .
$$

The substitution of $\tilde{u}$ from (5) in the above equation leads to (for details see Appendix A),

$$
\tilde{R}_{i j}(r)=\frac{1}{l^{2}} R_{i j} *\left(b_{I} \star b_{J}\right),
$$

where, $b_{I}$ and $b_{J}$ refer to the filters for $u_{i}$ and $u_{j}$, respectively.\|

Following the usual definition, spectrum $\left(\phi_{i j}\right)$ is defined as the Fourier transform of $R_{i j}$, i.e., $\mathcal{F}\left[R_{i j}\right]=\phi_{i j}$ (for the definition of Fourier transform and inverse used here, see eqn (34) in Appendix A), which for the filtered field becomes,

$$
\tilde{\phi}_{i j}(k)=\frac{2 \pi}{l^{2}} \phi_{i j} \mathcal{F}\left[b_{I} \star b_{J}\right] \text {, }
$$

after employing eqn (35), where $k$ is the wavenumber in the Fourier space corresponding to $r$ in the real space. Further simplifying the above expression using eqn (36) leads to the equation for the filtered spectrum as,

$$
\tilde{\phi}_{i j}(k)=\frac{4 \pi^{2}}{l^{2}} \phi_{i j} \hat{b}_{I}^{*} \hat{b}_{J}
$$

|| After the derivation of eqn (8) it came to our attention that a similar expression has been derived by Uberoi and Kovasznay [1953]; however, the main difference is that their expression has $\left(b_{J} \star b_{I}\right)$ while ours has $\left(b_{I} \star b_{J}\right)$. The difference between derivations starts at eqn (2) where instead of taking the filtered signal as a 'convolution' of the filter and the un-filtered signal, they start with a 'correlation', which implies the appropriate assignment of meanings to the filters. The results are equivalent if the filter functions are symmetric or they are the same for both velocity components, which is usually the case. 

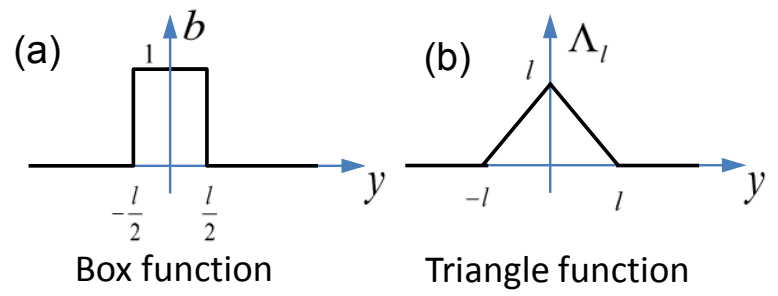

Figure 6. Schematic of two functions employed in the text. (a) Box function (with the maximum value equal to 1 ). (b) Triangle function (with the maximum value equal to $l$ ).

where, $\hat{b}_{I}$ and $\hat{b}_{J}$ are Fourier transforms of $b_{I}$ and $b_{J}$, respectively, and the superscript, * represents complex conjugate.

Of all the statistical quantities of turbulence that is measured, variance of the velocity field (e.g., $u_{r m s}^{2}$ for streamwise velocity) has been the primary object in considering the effect of spatial averaging [e.g., Moin and Spalart, 1987, Suzuki and Kasagi, 1992, Chin et al., 2009, Segalini et al., 2011a]. In terms of the above definitions, the variance of the streamwise velocity or the normal stress in direction 1 becomes,

$$
\tilde{u}_{r m s}^{2}=\tilde{R}_{11}(r=0)=\int_{-\infty}^{\infty} \tilde{\phi}_{11}(k) \mathrm{d} k
$$

and similar expressions hold for $\tilde{v}_{r m s}^{2}$ and $\tilde{w}_{r m s}^{2}$.

\subsection{Statistics from a single hot-wire with a box-filter}

Assuming that the hot-wire is sufficiently long, or that the $l / d$ is greater than 200 , where $d$ is the diameter of the wire, the temperature profile can be approximated to be a constant over the entire length of the wire. In this scenario, the filter function can be assumed to be of a 'box' type [e.g., Wyngaard, 1968, Chin et al., 2009]. For smaller $l / d$, end-conduction effects become important [e.g., Hultmark et al., 2011]. A schematic of this filter is given in figure 6(a) with an area equal to $l$. In such a case, since the hot-wire measures only $u$-velocity, eqn (8) for the expression of the filtered correlation can be simplified to,

$$
\tilde{R}_{11}\left(r_{2}\right)=\frac{1}{l^{2}} R_{11} * \wedge_{l}
$$

where, $\wedge_{l}$ is the triangle function, which is the correlation between two box functions and are shown schematically in figure 6(b). Furthermore, the spectrum of the filtered signal becomes,

$$
\tilde{\phi}_{11}\left(k_{2}\right)=\phi_{11} \operatorname{sinc}^{2}\left(k_{2} l / 2\right),
$$

where, the function $\operatorname{sinc}(x)$ is equal to $\sin (x) / x$, and appears in eqn (13) because the Fourier transform of the box function is $(1 /(2 \pi)) \sin \left(k_{2} l / 2\right) /\left(k_{2} / 2\right)$. Equation (13) has been derived by Wyngaard [1968] using Fourier-Stieltjes expansion for the work on HIT. However, the derivation given here is more general, where we start from eqn (10) valid 
for any filter, and only later consider the box-filter. As will be seen shortly, the equation in real space, i.e. the correlation in (8) or (12) is more significant for understanding and modelling the physical process of averaging than its counterpart (10) or (13). Finally, the expression for averaged $\tilde{u}_{r m s}^{2}$ can be obtained from (12) by substituting $r_{2}=0$ and employing the fact that $R_{11}(s)=R_{11}(-s)$ due to statistical stationary nature of the flow. Starting with,

$$
\tilde{R}_{11}\left(r_{2}\right)=\frac{1}{l^{2}} \int_{-l}^{l} R_{11}\left(r_{2}-s\right) \cdot(l-|s|) \mathrm{d} s
$$

leads to,

$$
\tilde{u}_{r m s}^{2}=\tilde{R}_{11}\left(r_{2}=0\right)=\frac{2}{l^{2}} \int_{0}^{l} R_{11}(s) \cdot(l-s) \mathrm{d} s,
$$

an equation first obtained by Dryden et al. [1937] and later by Frenkiel [1954] employing different techniques; however, without connecting it with (12).

The correctness of the expressions (12), (13) and (14) can be asserted by using the DNS database at $R e_{\tau}=934$. Since we are currently interested only in a single-wire in the homogeneous $x y$-plane, any location of $z^{+}$would suffice. We select $z^{+} \approx 15$ where the $u_{r m s}^{2}$ has a maximum and the filtering effects are most prominent. All the other $z^{+}$locations are independent homogeneous planes (as far as the single hot-wire is concerned) and can be tackled similarly, results of which will be presented later.

Figure $7(\mathrm{a})$ shows the attenuation of $u_{r m s}^{2+}$ at $z^{+} \approx 15$ for the box-filters with length typical of the hot-wires commonly used. The filled (black) symbol shows the resolution of the DNS whereas empty symbols are those which are calculated using a box-filter on the DNS field. The dashed (blue) line is the one calculated using (14) and the solid (red) line corresponds to its spectral counterpart (c.f., eqns 11 and 13). The un-averaged spectrum is calculated by averaging spectrum obtained from multiple DNS fields at $z^{+} \approx 15$ and correlation from its Fourier transform. Figures $7(\mathrm{~b})$ and (c) show the correlation in the spanwise direction, and the pre-multiplied spectrum plotted against the spanwise wavelength, $2 \pi / k_{2}^{+}$, respectively. The DNS results of the un-averaged distribution are shown in solid (black) lines, and the box-filtered results with $l^{+}=19$ by dashed (blue) lines and with $l^{+}=57$ by dashed dotted (red) lines. The symbols correspond to equations (12) and (13) in figures $7(\mathrm{~b})$ and (c), respectively. Note that, in figure $7(\mathrm{~b})$ the $\tilde{R}_{11}^{+}$is not normalized with its value at $r_{2}^{+}=0, u^{2^{+}}$. The result is that all three methods of averaging (actual box filtering on the DNS field, using the un-averaged correlation function and spectrum) match each other. This is not surprising and indicates that expressions (12), (13) and (14) are exact for box-filtering.

However, it is of practical interest to note that if the averaged correlation or spectrum is obtained from a hot-wire, the relations (12) and (13) can be inverted to find the corresponding un-averaged distributions from the averaged ones. Even though figures 7(b) and (c) can be related to each other through the Fourier transform, they show interesting features by themselves. Figure 7(c) indicates that smaller wavelengths are attenuated much more than larger ones, corresponding to the $\operatorname{sinc}^{2}\left(k_{2} l / 2\right)$ function in 

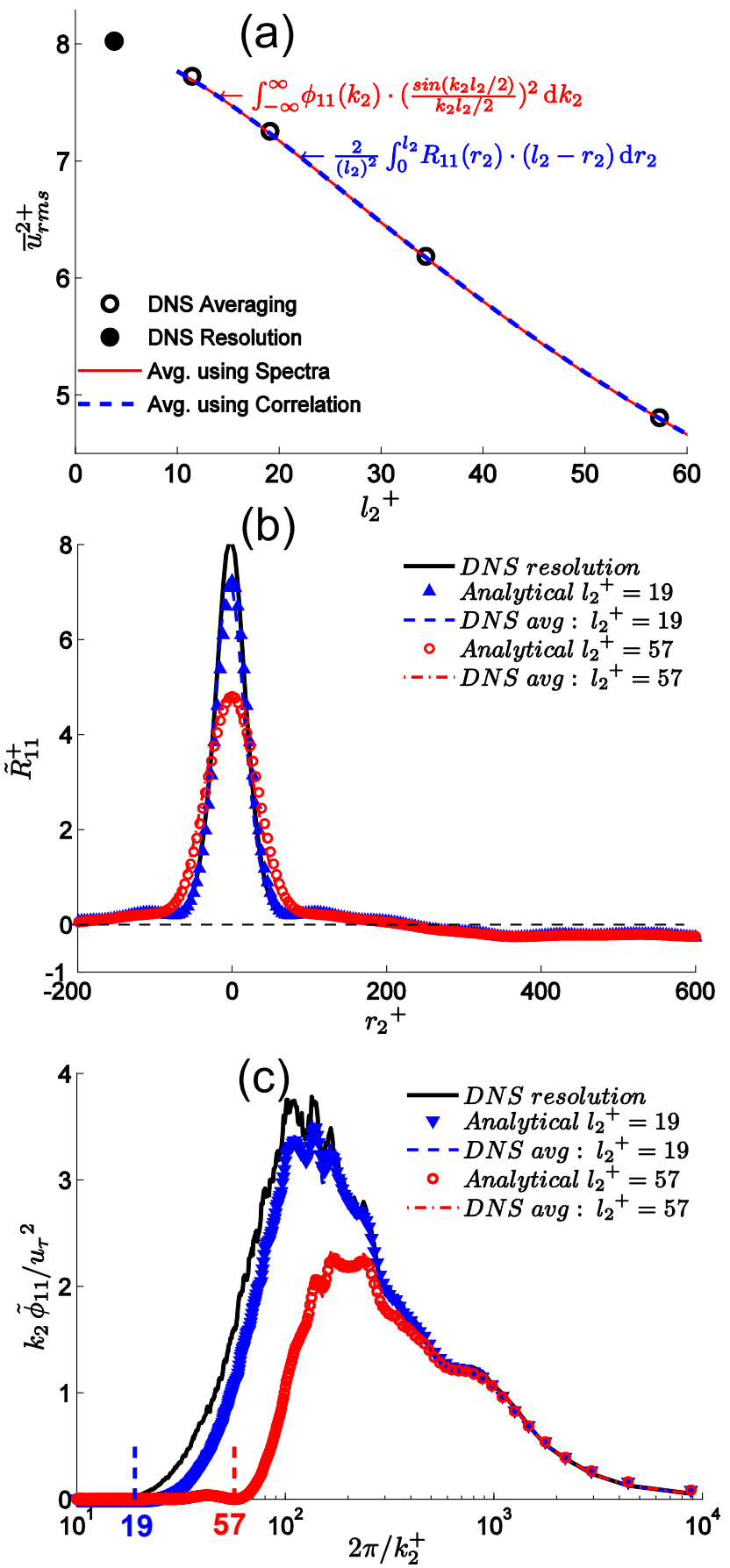

Figure 7. Averaging effect for $z^{+}=15$ at $R e_{\tau}=934$ using DNS database for various $l_{2}^{+}$. (a) Attenuation in $u_{r m s}^{2+}$ with three different methods, namely, actual averaging on the velocity field (symbols), using the spectrum (solid, red line) and with the correlation function (dashes, blue line), c.f. eqn (11). (b) Attenuation in correlation $R_{11}^{+}$for $l_{2}^{+}=19$ and 57, calculated with the DNS database (solid line) and using the exact expression, (12). (c) Attenuation in the spectrum $\phi_{11}$ for $l_{2}^{+}=19$ and 57, calculated with the DNS database (solid line) and using the exact expression, (13). The two vertical lines at 19 and 57 indicate the lower cut-off wavelengths, $2 \pi / k_{2}^{+}$. However, note that attenuation is still significant even for higher wavelengths. 
(13). It shows that wavelengths smaller than $l=2 \pi / k_{2}$ (the first zero of $\operatorname{sinc}^{2}\left(k_{2} l / 2\right)$ ) are almost completely attenuated and the attenuation decreases with increasing wavelength with zero attenuation occurring only at infinite wavelength. This shows that attenuation is not localized in spectral space even though the cut-off point can be approximated by $l=2 \pi / k_{2}$ for a hot-wire of length $l$. On the other hand, expression (14) shows that $u_{r m s}^{2+}$ depends only on $R_{11}$ in the range 0 to $l$. This 'localization effect' suggests that $R_{11}$ should be the quantity to be focused upon to understand and model the spatial averaging effects. A starting point in this direction is taken up next.

\subsection{A simple model for the spanwise correlation and some consequences}

The unfiltered distribution (at the DNS resolution) of $R_{11}$ was shown in figure $7(\mathrm{~b})$; an enlarged view of the same, normalized with the value at $r_{2}=0$ is presented in figure 8 with empty symbols connected by solid lines. The correlation attains its second maxima (after the origin) near $r_{2}^{+} \approx 100$, and oscillates before approaching the zero value due to the statistical stationary turbulent flow. The particular shape of the correlation is of interest because of (14) wherein $R_{11}$ in the range 0 to $l$ governs the spatial filtering of $u_{r m s}^{2}$. The physical reason of the dip in $R_{11}^{+}$is the well known near-wall streaky behavior of the turbulent boundary layer.

It is not difficult to model this small region of the correlation governed by the nearwall streaks, which does not change with Reynolds number in viscous units. The two most common models for the normalized $R_{11}$, denoted by $R_{N}$ are presented in figure 8 . The first is the decaying exponential type, $R_{N}=\mathrm{e}^{-r_{2} / \lambda_{1}}$, where $\lambda_{1}$ is a constant found from fitting the data up to $r_{2}^{+}=100$. The second is the Gaussian type model,

$$
R_{N}\left(r_{2}\right)=\mathrm{e}^{-r_{2}^{2} / \lambda^{2}},
$$

where, $\lambda^{+}=26$ is the constant found from fitting to the DNS curve at $z^{+}=15$. It is obvious that the Gaussian curve is a better representation of the correlation near the origin. In fact, $\lambda^{+}=26$ is about $1 / 4$ times the streak spacing and it will be shown later that 4 times the streamwise length scale for a similar correlation model in the $x$-direction will correspond closely to the streamwise length of the near-wall streaks of 1000 wall-units. In short, the near-wall streaks dictate the correlation function close to the wall, especially near $r_{2}^{+}=0$.

Considering (15) as the normalised model for spanwise correlation of streamwise velocity, the corresponding spectrum can be found from its Fourier transform $\left(\phi_{N}=\right.$ $\left.\mathcal{F}\left[R_{N}\right]\right)$ as,

$$
\phi_{N}\left(k_{2}\right)=\frac{1}{2 \pi} \sqrt{\pi} \lambda \mathrm{e}^{-\left(k_{2} \lambda / 2\right)^{2}}
$$

The filtered correlation $\left(\tilde{R}_{N}\right)$ can be obtained by substituting (15) into (12) and evaluating the convolution with $\wedge_{l}$ as,

$$
\tilde{R}_{N}\left(r_{2}\right)=\frac{\lambda^{2}}{2 l^{2}}\left[\frac { \sqrt { \pi } } { \lambda } \left\{\left(r_{2}+l\right) \operatorname{erf}\left(\frac{r_{2}+l}{\lambda}\right)\right.\right.
$$




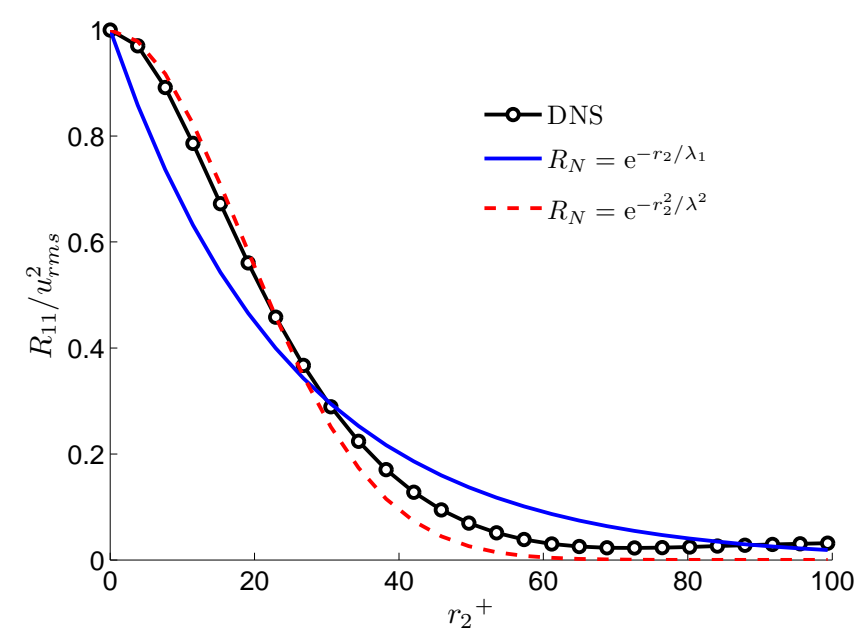

Figure 8. Two different models for the normalized correlation function up to $r_{2}^{+}=100$.

$$
\begin{aligned}
& \left.-2 r_{2} \operatorname{erf}\left(\frac{r_{2}}{\lambda}\right)+\left(r_{2}-l\right) \operatorname{erf}\left(\frac{r_{2}-l}{\lambda}\right)\right\} \\
& \left.+\left\{\mathrm{e}^{-\left(r_{2}-l\right)^{2} / \lambda^{2}}-\mathrm{e}^{-\left(r_{2}+l\right)^{2} / \lambda^{2}}-2 \mathrm{e}^{-r_{2}^{2} / \lambda^{2}}\right\}\right],
\end{aligned}
$$

where, $\operatorname{erf}(x)=2 / \sqrt{\pi} \int_{0}^{x} \mathrm{e}^{-s^{2}} \mathrm{~d} s$, is the standard error function. The corresponding averaged spectrum is,

$$
\tilde{\phi}_{N}\left(k_{2}\right)=\frac{1}{2 \pi} \sqrt{\pi} \lambda \mathrm{e}^{-\left(k_{2} \lambda / 2\right)^{2}} \operatorname{sinc}^{2}\left(k_{2} l / 2\right)
$$

Furthermore, the attenuation in $u_{r m s}^{2}$ can be found from (17) by substituting $r_{2}=0$,

$$
\frac{\tilde{u}_{r m s}^{2}}{u_{r m s}^{2}}=\tilde{R}_{N}\left(r_{2}=0\right)=\frac{\lambda^{2}}{l^{2}}\left[l \frac{\sqrt{\pi}}{\lambda} \operatorname{erf}\left(\frac{l}{\lambda}\right)+\mathrm{e}^{-l^{2} / \lambda^{2}}-1\right] .
$$

It is noted that the form of the above equation is also obtained by Segalini et al. [2011b], however, starting from (14) and considering $\lambda$ as the 'Taylor micro-scale' unlike the case considered here. The issue of relevant length scales for averaging in wall-turbulence will be discussed later.

Figure 9 shows a comparison between the DNS box-averaging in symbols and the estimations from the model (i.e. eqn 19) in solid line. The prediction is reasonably good due to the correct modeling of the correlation which in turn governs the attenuation in $u_{r m s}^{2}$. Note that eqn (19) is only a function of $l / \lambda$ and it can be concluded that the correct parameter to study attenuation in $u_{r m s}^{2}$ is $l / \lambda$ and not $l$ alone. The predication by (19) should be contrasted with that of fitting the DNS averaged points by Chin et al. [2009] using a third order polynomial with $l^{+}$as the parameter. The significance of $l / \lambda$ is that as long as the inner-scaled streak spacing does not change (which is known to be quite a robust phenomenon across a range of $R e$ ), the value of $\lambda^{+}=26$ does not change, however, as soon as the structures in the flow change (for example by moving away from 


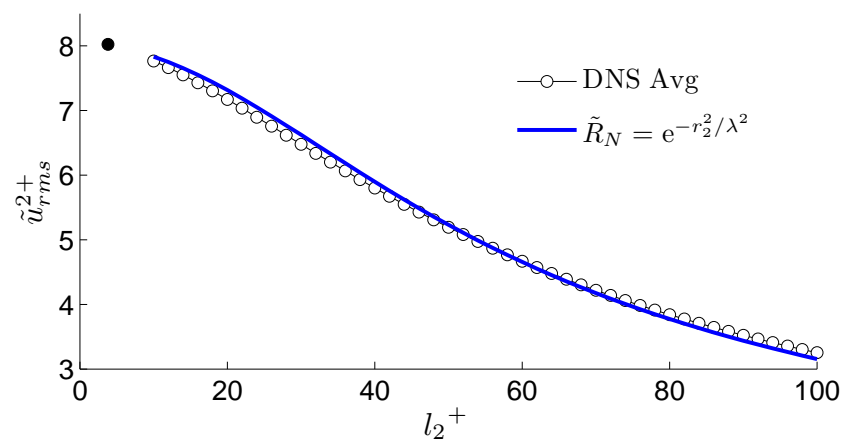

Figure 9. The attenuation in $u_{r m s}^{2}$ calculated by DNS and that estimated from the model using (19).

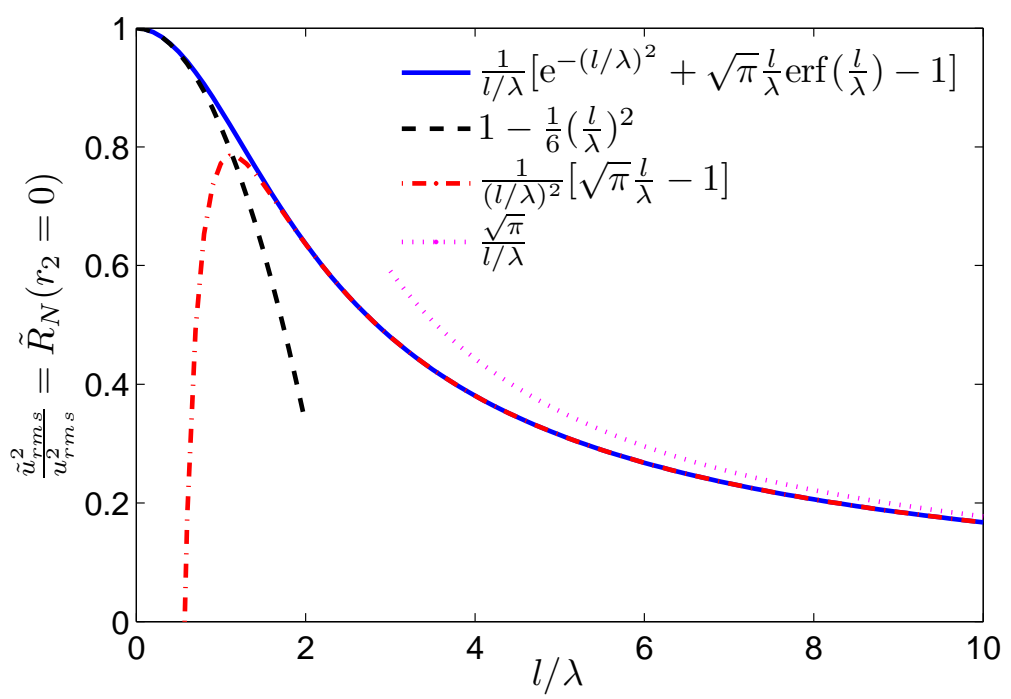

Figure 10. Various approximations for the expression on the extreme right of (19) for different limits of $l / \lambda$.

the wall in TBL, or in a flow with no wall) the value of $\lambda$ will change. Nevertheless, (19) will continue to hold but $l / \lambda$ is now unknown.

It is interesting to note that eqn (19) takes different forms as the parameter $l / \lambda$ approaches different limits. These limits not only simplify the expression but also relate to some of the expressions derived by earlier researchers. In the limit of $l / \lambda$ approaching zero,

$$
\lim _{(l / \lambda) \rightarrow 0} \tilde{R}_{N}\left(r_{2}=0\right)=1-\frac{1}{6}\left(\frac{l}{\lambda}\right)^{2} .
$$

This is the expression first found by Frenkiel [1949] considering $\lambda$ as the 'Taylor microscale' (unlike here) and later criticized by Corrsin and Kovasznay [1949] as unreliable for large $l / \lambda$. The correct expression for the limit of $l / \lambda$ approaching large values is,

$$
\lim _{(l / \lambda) \rightarrow \infty} \tilde{R}_{N}\left(r_{2}=0\right)=\sqrt{\pi}\left(\frac{\lambda}{l}\right)
$$



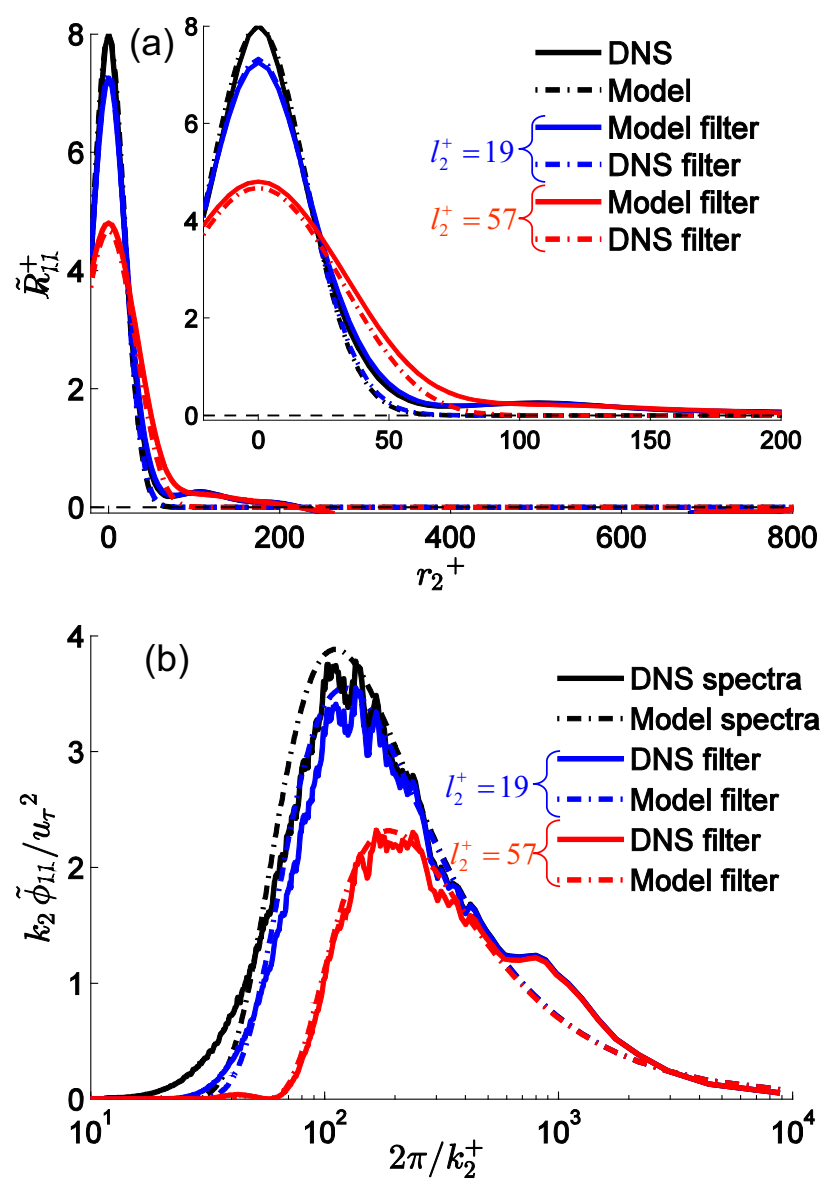

Figure 11. Attenuation in the correlation and spectrum, from DNS and the model. (a) Averaging effect on $R_{11}$ from DNS (solid line) and (17). (b) Averaging effect on premultiplied spectrum $k_{2} \phi_{11}$ from DNS (solid lines) and (18).

Furthermore, a higher order approximation to this limit is $1 /(l / \lambda)^{2}[\sqrt{\pi} /(l / \lambda)-1]$. All the three simplified expressions are plotted in figure 10 for comparison. It can be observed that different expressions are valid (i.e. matches the eqn 19) in the appropriate limits of the parameter $l / \lambda$. However, the most interesting scenarios from the practical use of single hot-wires are when $l \approx \lambda$. Unfortunately, in this situation, none of the three cases is a good representative of (19).

It is worthwhile to test the capability of the model to estimate the entire filtered correlation and spectrum in the spanwise direction, considering that the model was derived by fitting to the actual correlation only up to $r_{2}^{+}=100$. Figure 11(a) shows the actual and two filtered correlations with $l^{+}=19$ and 57 from DNS and the corresponding un-filtered correlations from (15) and filtered ones from (17). The inset shows an enlarged view. The model equation follows the filtered correlations quite well in the region close to the origin (i.e. the inset), however, the model unfiltered/filtered correlation drops to zero and remains there for increasing $r_{2}^{+}$whereas the actual correlation exhibits a much more complicated distribution. A similar plot for the premultiplied spectrum $\left(=k_{2} \phi_{11} / u_{\tau}^{2}\right)$ is shown in figure 11(b) which again shows the good 


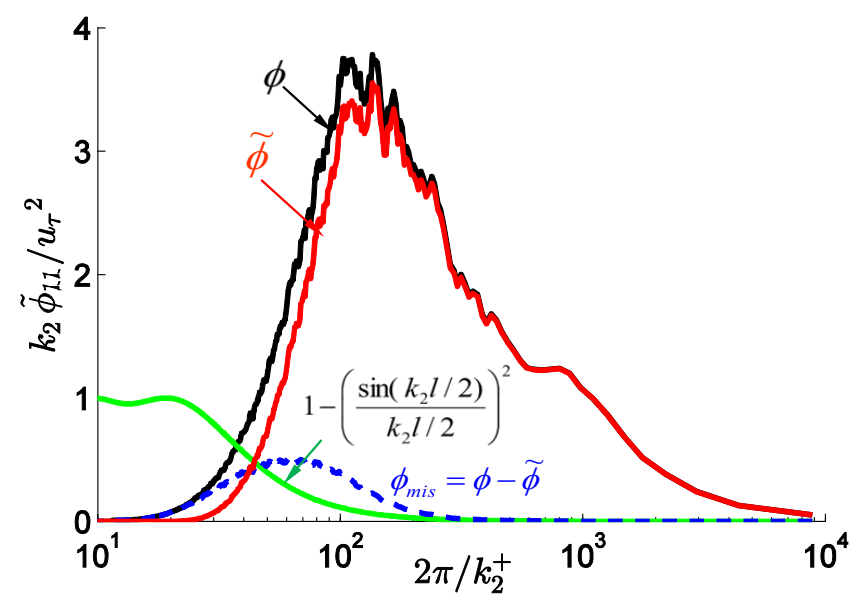

Figure 12. An example of the missing spanwise spectrum from DNS at $l^{+}=19$.

comparison between the DNS data (both unfiltered and filtered) in solid lines and the model from (18) in dashed-dotted lines. The peak at about 100 viscous units is predicted closely as well as the overall shape of the spectrum. However, the model exhibits some loss of energy at large wavelengths.

There have been efforts in the past to develop models for the 'missing spectra', especially by Chin et al. [2009, 2011]. The missing spectrum, $\phi_{m i s}$ is the difference between the unfiltered and the filtered spectrum, $\phi_{m i s}=\phi-\tilde{\phi}$. The model for $\phi_{m i s}$ has been found from observation to be of a Gaussian type. Here it is of interest to see if the correlation model of (15) can be used to understand this behavior of $\phi_{\text {mis }}$. Figure 12 shows an example of the filtered signal $\tilde{\phi}$ where $l^{+}=19$ and the difference, $\phi_{\text {mis }}$. Indeed, the distribution of $\phi_{m i s}$ does look like a Gaussian. It can be observed that, using (13) we can write,

$$
\phi_{N_{m i s}}=\phi_{N}-\tilde{\phi}_{N}=\phi_{N}\left(1-\operatorname{sinc}^{2}\left(k_{2} l / 2\right)\right) .
$$

Thus, $\phi_{m i s}$ is the product of the un-filtered $\phi$ and the function, $\left(1-\operatorname{sinc}^{2}\left(k_{2} l / 2\right)\right)$, which is also shown in figure 12 . We can proceed further by employing the model spectrum of (16), which leads to,

$$
\phi_{N_{m i s}} \approx \frac{1}{2 \pi} \sqrt{\pi} \lambda \mathrm{e}^{-\left(k_{2} \lambda / 2\right)^{2}}\left(1-\operatorname{sinc}^{2}\left(k_{2} l / 2\right)\right)
$$

where the approximation $\operatorname{sign}(\approx)$ is used to indicate that the expression for $\phi_{m i s}$ is using the model equation. The effectiveness of this prediction is presented in figures 13(a) and (b) corresponding to averaging with $l^{+}=19$ and 57, respectively. As observed from the figure, the predictions of (23) matches reasonably well with that obtained from DNS (indicated in the figure by ' $\phi_{m i s}$ ') for both the averaging conditions. It is now evident that even though the shape of $\phi_{m i s}$ resembles a Gaussian curve, it is probably a more complicated function approximated by (23).

Next, we turn to the hot-wire averaging effects on the streamwise spectrum. The un-averaged pre-multiplied streamwise spectrum (normalized with plus units) is shown 

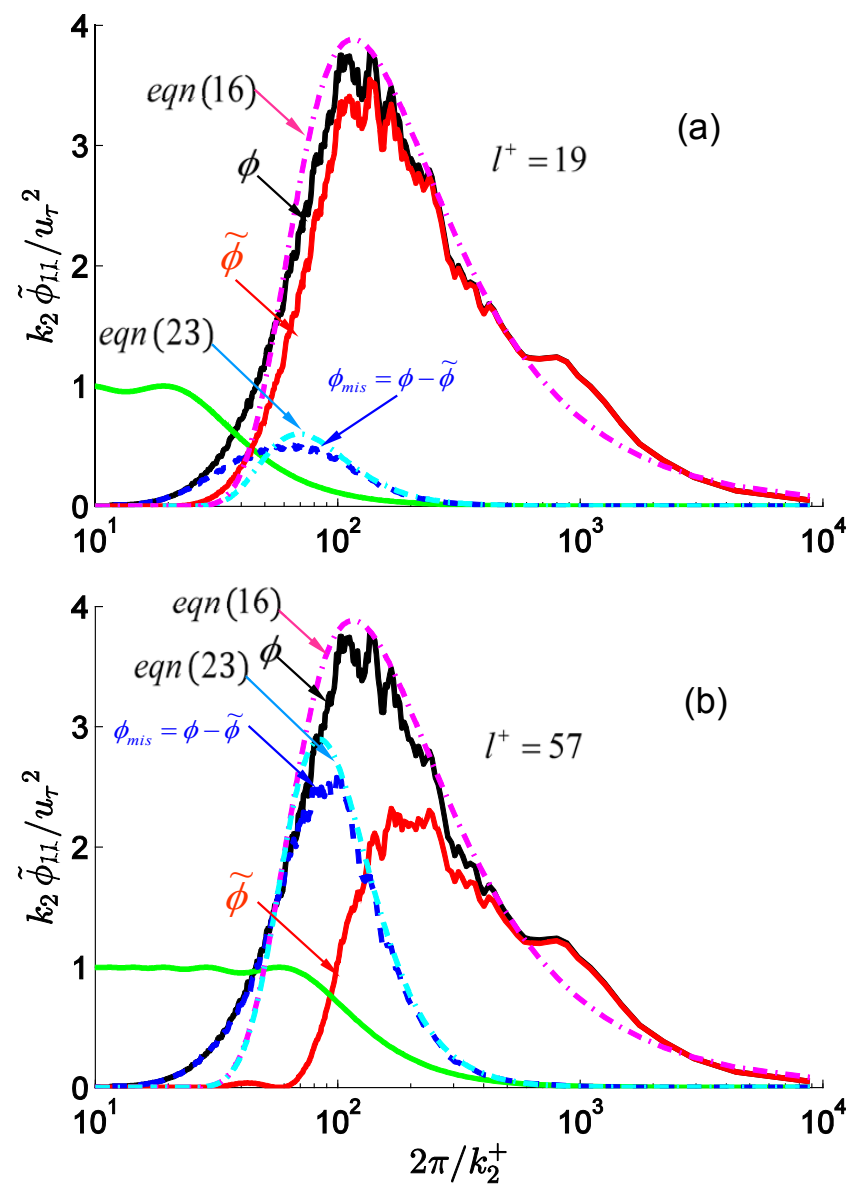

Figure 13. Predictions of the missing spectrum (plotted in pre-multiplied form) and comparison with the DNS. (a) $l^{+}=19$. (b) $l^{+}=57$.

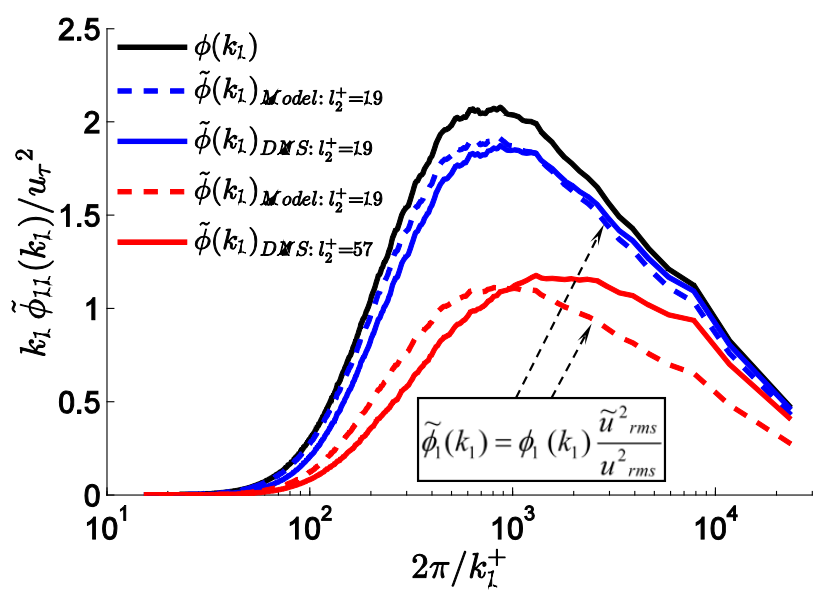

Figure 14. Streamwise spectrum from DNS (solid lines) and the model (dashed lines, from eqn (28)) for two filtering lengths, $l^{+}=19$ and 57 . 
in figure 14 by a solid (black) line. Note that the spectrum peaks at a wavelength of about 1000 viscous units, which has been found to be the average streamwise length of the near-wall streaks. The averaged spectrum for $l^{+}=19$ and 57 are also shown with solid lines (blue and red, respectively). The attenuation pattern is very different from that of the spanwise spectrum (c.f. figure 7 or 11). In the spanwise spectrum it was observed that the attenuation is highly dependent on the wavelength $\left(2 \pi / k_{2}\right)$, with smaller wavelengths (depending on $l / \lambda$ ) almost completely attenuated and larger ones much less. For streamwise spectrum the smaller wavelengths are attenuated more than the large ones (and the explanation is not very different than that given for the spanwise case), however, the attenuation is more broadband (see for example $l^{+}=57$ case in figure 14). Some understanding of this behavior can be obtained by considering the equations for streamwise spectrum (which for the sake of simplicity is assumed to be normalized to have variance of unity), and can be written as,

$$
\phi_{11}\left(k_{1}\right)=\int \phi_{11}^{2 D}\left(k_{1}, k_{2}\right) \mathrm{d} k_{2}
$$

where, $\phi_{11}^{2 D}\left(k_{1}, k_{2}\right)$ is the two-dimensional spectrum and the integration is over the whole domain of $k_{2}$. Furthermore, the average streamwise spectrum becomes,

$$
\tilde{\phi}_{11}\left(k_{1}\right)=\int \tilde{\phi}_{11}^{2 D}\left(k_{1}, k_{2}\right) \mathrm{d} k_{2} \text {. }
$$

Since the single hot-wire is averaging in the spanwise direction,

$$
\tilde{\phi}_{11}\left(k_{1}\right)=\int \phi_{11}^{2 D}\left(k_{1}, k_{2}\right) \operatorname{sinc}^{2}\left(k_{2} l / 2\right) \mathrm{d} k_{2} .
$$

The difficulty for predicting $\tilde{\phi}_{11}\left(k_{1}\right)$ is clear now; one has to know the distribution of $\phi_{11}^{2 D}\left(k_{1}, k_{2}\right)$. This is the reason that Chin et al. [2009] employed their Gaussian type fitting function to predict back $\tilde{\phi}_{11}\left(k_{1}\right)$. To make any further progress one has to adopt some model for $\phi_{11}^{2 D}\left(k_{1}, k_{2}\right)$. Again, to begin with, we resort to a crude model by writing the $2 \mathrm{D}$ function as a product of individual spectrum in $k_{1}$ and $k_{2}$,

$$
\phi_{11}^{2 D}\left(k_{1}, k_{2}\right) \approx \phi_{11}\left(k_{1}\right) \cdot \phi_{11}\left(k_{2}\right),
$$

and, this allows us to take $\phi_{11}\left(k_{1}\right)$ out of the integral in (26). Alongside, $\phi_{11}\left(k_{2}\right)$ can be substituted with the model spectrum employed previously given in (16), which leads to,

$$
\begin{aligned}
\left.\tilde{\phi}_{11}\left(k_{1}\right)\right) & \approx \phi_{11}\left(k_{1}\right) \int \frac{1}{2 \pi} \sqrt{\pi} \lambda \mathrm{e}^{-\left(k_{2} \lambda / 2\right)^{2}} \operatorname{sinc}^{2}\left(k_{2} l / 2\right) \mathrm{d} k_{2} \\
& =\phi_{11}\left(k_{1}\right) \frac{\lambda^{2}}{l^{2}}\left[l \frac{\sqrt{\pi}}{\lambda} \operatorname{erf}\left(\frac{l}{\lambda}\right)+\mathrm{e}^{-l^{2} / \lambda^{2}}-1\right] \\
& =\phi_{11}\left(k_{1}\right) \frac{\tilde{u}_{r m s}^{2}}{u_{r m s}^{2}},
\end{aligned}
$$

where, in the first equation the limits are from $-\infty$ to $\infty$ and (19). It shows that within the assumption of the model (27) the averaged streamwise spectrum is simply a constant in $k_{1}$ (depending on $l / \lambda$ ) times the un-averaged spectrum, which is only partially correct. Figure 14 shows (28) with dotted lines for two hot-wire lengths of $l^{+}=19$ and 57 . 
The estimations are not as good as they are for the spanwise spectrum due the crude model in (27). Anyhow it clarifies to some extent why there is less variation of the averaged streamwise spectrum with the wavelength compared to the averaged spanwise one. Given that the hot-wire only measures $\tilde{\phi}_{11}\left(k_{1}\right)$, multiplication by the factor in $(28)$ would definitely give a close approximation to the actual un-averaged spectrum. As an example, for standard hot-wires, $l^{+}=19$ is a good estimate, and the prediction by (28) for this case in figure 14 is not bad. Note that if we build a model for $R_{11}^{2 D}\left(r_{1}, r_{2}\right)$ over a small region, it is not difficult to recover the un-averaged spectrum from the averaged ones; however, this will take us too far into correlation modelling, beyond the scope of the present work.

\subsection{Effect of wallnormal distance (z)}

Thus far all the analysis is concerned with a single $z$-location, namely $z^{+}=15$. This analysis can be extended to other $z$-locations in a similar manner. The model for correlation (15) with $\lambda^{+}=26$ predicts the attenuation in $u_{r m s}^{2+}$ quite well. However, the model (15) as well as the particular value of $\lambda$ would not be expected to be invariant with the $z$-location, since it is well known that the correlation function varies with $z$. Knowledge of the correlation function at different $z$-locations would render the problem of finding the filtered normal stress, $\tilde{u}_{r m s}^{2}$, solved. However, the entire correlation function need not be known. As discussed above, the function from $(0-l)$ (where $l$ is length of the hot-wire) is sufficient to predict the actual $u_{r m s}^{2}$. To this end, figure 15 shows the correlations $\Phi$ normalised with variance (such that the value at $r_{2}^{+}=0$ is unity) for different $z$-locations at $R e_{\tau}=934$. The inset shows a zoomed view of the correlations close to the origin. Close to the wall the correlations are dominated by the near-wall streaks whereas further out in the boundary-layer, structures of the order of the boundary-layer thickness ( $\delta$ or $R e_{\tau}$ in plus units) control the correlation functions. The correlation remains close to zero beyond $r_{2}^{+} \approx 2000$ and asymptotes to zero in the limit.

To understand the attenuation in $u_{r m s}^{2}$ only correlations up to $r_{2}^{+}=100$ are considered (c.f. figure 15-inset) and the model (15) can be fitted to the correlations at various $z$-locations to find the characteristic length scale, $\lambda$. This is presented in figure 16 as a function of $z^{+}$. The value of $\lambda^{+}$remains constant (at approximately 26 as already found earlier) for small $z^{+}$consistent with the near-wall streaks and steadily increases until $z / \delta \approx 0.5$, and thereafter remains more or less constant.

The effect of $z$-location of the attenuation in $u_{r m s}^{2}$ is presented in figure 17(a), wherein the solid (black) line shows the un-attenuated (at the resolution of DNS) variance and symbols correspond to that obtained from the filtering using the DNS database. Given the values of $\lambda$ presented in figure 16, the model (15) can be used to find the filtered distribution using (17). The dashed lines in figure 17(a) are calculations

ฯ The correlations presented in this and the subsequent sections are calculated from the spectra available online at "http://torroja.dmt.upm.es/channels/". 


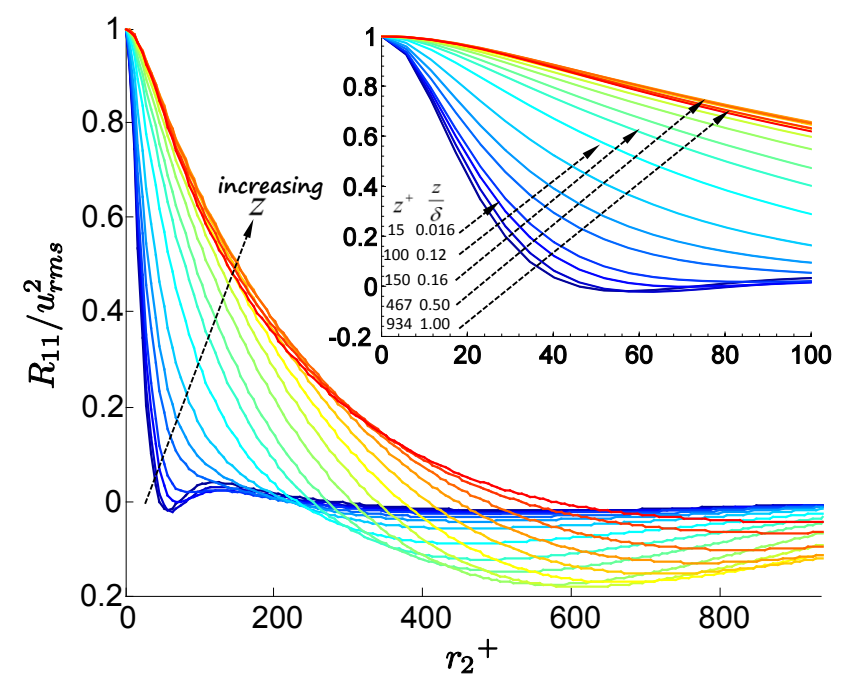

Figure 15. Distribution of the normalized correlation $R_{11} / u_{r m s}^{2}$ along spanwise direction, $r_{2}^{+}$for $R e_{\tau}=934$, with $z^{+}=5,10,15,19,30,40,60,100,149,198$, $279,371,467,562,656,744,835$ and 934 . The inset shows an expanded view, with some $z$-locations indicated on the figure itself.

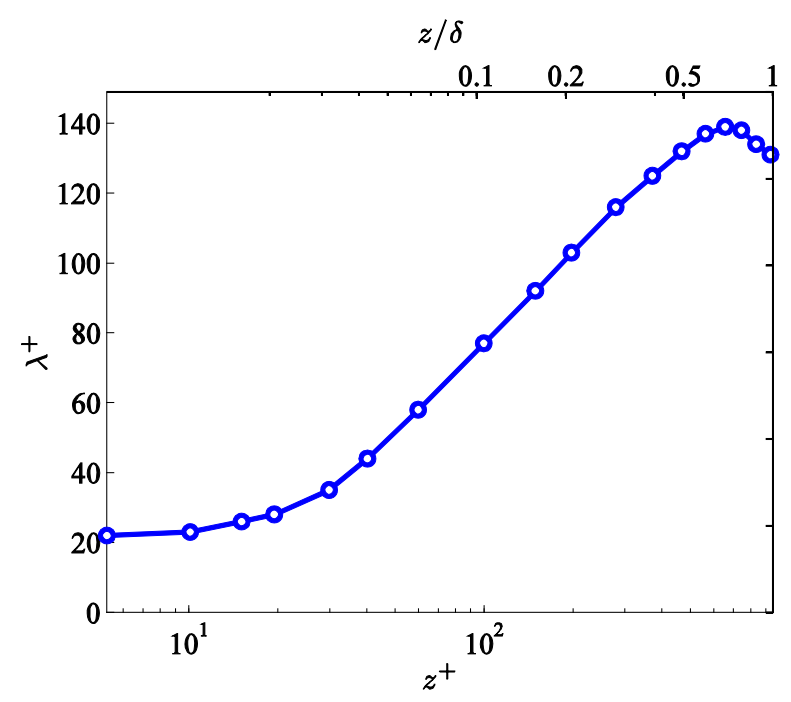

Figure 16. Distribution of $\lambda^{+}$defined in (15) with wallnormal distance, $z^{+}$, obtained from the data in the inset of figure 15, i.e., up to $r_{2}^{+}=100$.

from the model (19) and the corresponding hot-wire lengths $\left(l^{+}\right)$are indicated in the figure. Similar calculations for varying $l^{+}$for five different $z$-locations are presented in 17 (b), with the corresponding values of $z^{+}$and $z / \delta$ indicated on the figure. The agreement between the model and the DNS calculations is seen to be good. This is not much of a surprise because the values of $\lambda$ were already calculated. The challenge would be to build a 'physically' reasonable model for $\lambda$ when $z$ varies. This will be one of the goals later in the paper where we also try to understand the effect of Reynolds number. At this point we shall digress a little to discuss the relevant length scales in averaging. 

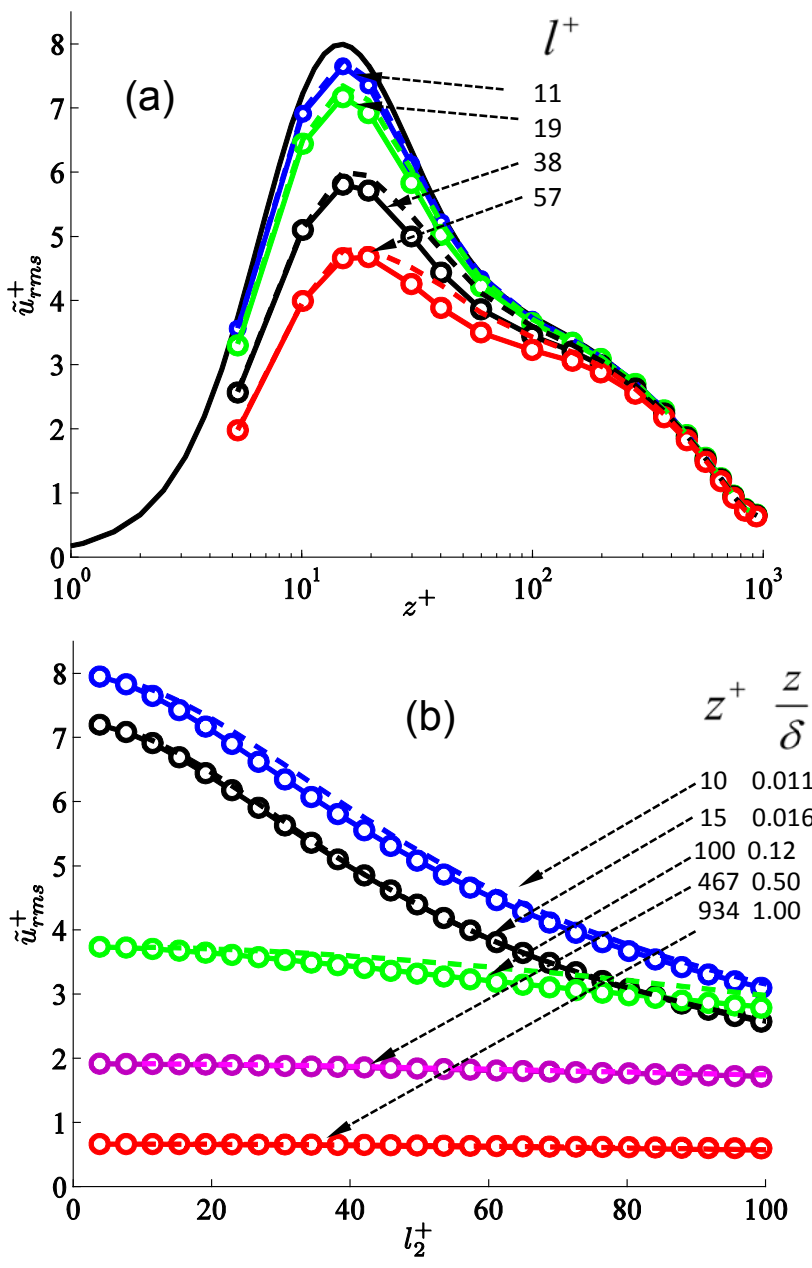

Figure 17. Comparison of model (19) in dashed lines, with the filtering results obtained from DNS in symbols at $R e_{\tau}=934$. (a) Variation of $u_{r m s}^{2+}$ with $z^{+}$for $l^{+}$of 11, 19, 38 and 57, as indicated in the figure. (b) Variation of $u_{r m s}^{2+}$ with $l^{+}$ for different wallnormal planes located at $z^{+}=10,15,100,467$ and 934, with the corresponding $z / \delta$ indicated in the figure.

\subsection{Relevant length scales for spatial averaging}

The two most commonly used length scales are the viscous (inner) units, $\nu / u_{\tau}$ and the Taylor micro-scale, say $\lambda_{T}$. Here we have introduced another length scale, the characteristic correlation length scale $\lambda$ through the model correlation function, which we believe to be the representative length scale for the averaging process. It is useful to understand which of the three length scales is most appropriate and why each has in the past been considered as probable candidates. On considering the viscous length scale, it is noted that $\nu / u_{\tau}$ is only relevant for 'wall-bounded flows', therefore inner units cannot in general be the correct length scale for hot-wire averaging (such as in free-shear flows). However, they have been found to be appropriate for wall-turbulence, especially close to the wall. The reason being that, as already shown, $\lambda$ scales with inner units close to the wall and therefore, $\lambda$ and $\nu / u_{\tau}$ differ only by a constant close to the wall for any 

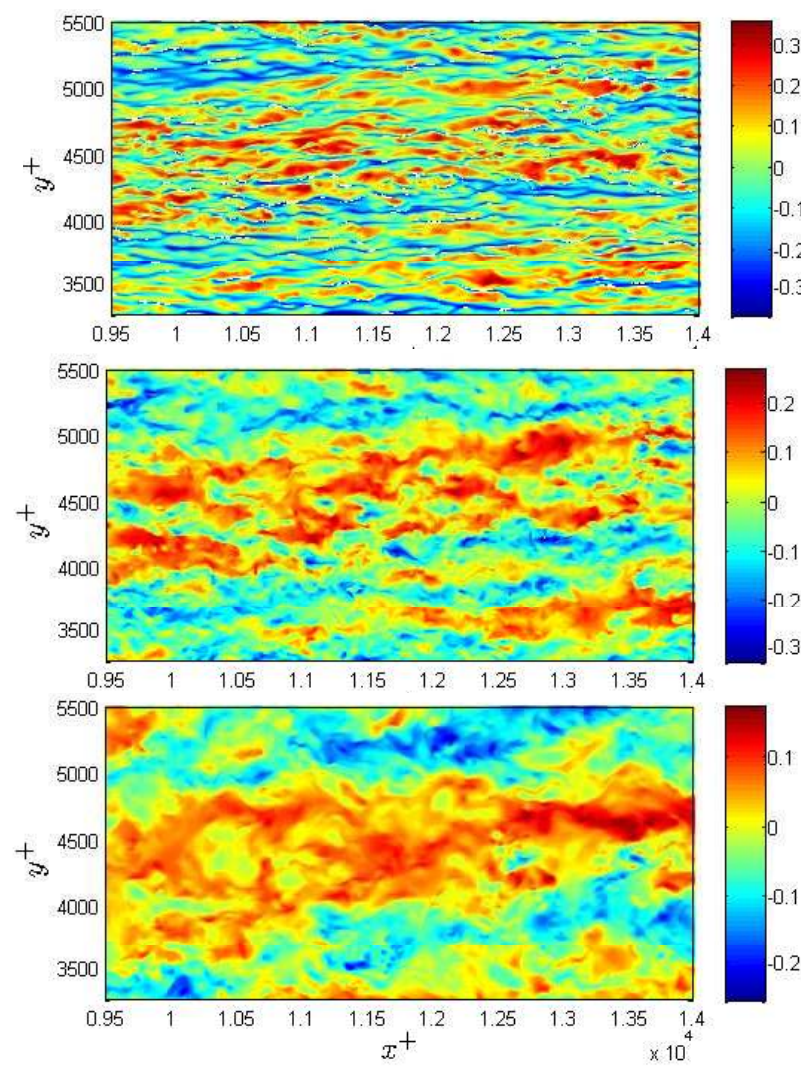

Figure 18. Instantaneous $u$ at $R e_{\tau}=934$ for a range of $4500^{+}(4.8 \delta)$ and $2250^{+}$ $(2.4 \delta)$ in $x$ and $y$-directions. (a) $z^{+}=15, z / \delta=0.016$. (b) $z^{+}=100, z / \delta=0.12$. (c) $z^{+}=467, z / \delta=0.5$.

Re. Thus there is no difference between $\lambda$ and inner units close to the wall, where the turbulent motions and therefore $\lambda$ scales on $\nu / u_{\tau}$.

To further illustrate this, consider figures 18(a), (b) and (c) corresponding to $u$ in the $x y$-plane at $z^{+}=15(z / \delta=0.016), z^{+}=100(z / \delta=0.12)$ and $z^{+}=467(z / \delta=0.5)$, respectively at a fixed $R e_{\tau}$ of 934 , i.e., a fixed value to $\nu / u_{\tau}$. It is immediately clear that a hot-wire placed in the flow field presented in the top panel will have a larger attenuation than the lower ones, because the spanwise scale of the structures in this figure are smaller. This implies that the attenuation changes with the structures or the correlation, characterized by $\lambda$, rather than $\nu / u_{\tau}$ which is the same for all three figures.

The Taylor micro-scale, $\lambda_{T}$, shares some features with $\lambda$ in the sense that both are related to the correlation function. However, since $\lambda_{T}$ depends only on the curvature at the origin, it is unable to capture critical features of the correlation function related to spatial averaging (primarily the minima in the $R$, unlike $\lambda$ ). This is precisely the reason that Segalini et al. [2011a] could not capture the attenuation with merely $\lambda_{T}$, and had to add multiple length scales to match the DNS calculations. Furthermore, physically $\lambda_{T}$ has little connection with spatial averaging; it instead provides a measure of dissipation in HIT, and the correspondence to spatial averaging (through the curvature of $R$ ) is a likely coincidence. Probably from a physical point of view, the integral length scales are 
more connected to the averaging issue (c.f., figure 23 in Appendix B) than $\lambda_{T}$, however, these too fall short of expectation close the wall. In short, the characteristic correlation length scale, $\lambda$ is the appropriate length scale for spatial averaging. This is abundantly evident from equation 19, which depends only upon $l / \lambda$.

Sometimes the Kolmogorov length scale $\eta$ is employed as the relevant length scale, most notably after the work of Wyngaard [1968], particularly for HIT. (Note that $\eta^{+}$ remains almost invariant with $R e$ in the near wall as well as in the log-region for wallturbulence; see, e.g., Yakhot et al. [2010], Marusic and Adrian [2013].) The usual procedure is to assume a form of energy spectrum (for example Pao's spectrum) and employ eqn (13) in conjunction with (11) to calculate $\tilde{u}_{r m s}^{2}$. However, spectral space is not as appropriate as real space (via eqn (14)) to model spatial averaging because in spectral space the exact form of spectrum is needed for 'all' wave-numbers, which is not easily possible (since all the wave-numbers are attenuated, except the trivial zero wave-number), whereas in real space the integral is limited only from 0 to $l$, and it is easier to represent $R_{11}$ in the short domain with a single length scale, $\lambda$.

\subsection{Effect of Reynolds number}

To investigate the effect of Reynolds number $(R e)$ on the spatial averaging, the DNS data from a channel flow simulation by del Álamo and Jiménez [2003] and del Álamo et al. [2003] at $R e=180,550,934$ and 2003 are employed. Since it is clear that correlations control the spatial averaging, figures 19 (a-d) show normalized correlations for varying $z$-locations for $R e=180,550,934$ and 2003, respectively, in a manner so as to reflect their inner and outer scaling. Each figure shows the distribution of correlations up to $r_{2}^{+}=R e_{\tau}$ (or $r_{2}=\delta$ ), whereas the inset figure shows the same up to $r_{2}^{+}=100$. Notice that for small $z$, i.e., close to the wall (c.f, the inset), the correlations for all Reynolds numbers have a minimum close to $r_{2}^{+} \approx 50$, dictated by the near wall streak spacing of $100^{+}$. This remains invariant with $R e$, and represents the inner viscous scaling. However, correlations far from the wall scale with the outer scaling, as evident from the main figures at all Re. This is not surprising because, like the mean flow and the turbulent stresses, close to the wall the correlations seem to scale with inner (or plus) variables and away from the wall they scale on $\delta$, the outer variable. This provides some hope for representing correlations in a $R e$ invariant form, which we can then utilize for the estimation of filtered quantities.

An example of such a model is developed in Appendix B along with an appropriate length scale and its comparison with other integral length scales. The model reads:

$$
R_{N}\left(r_{2}\right)=\mathrm{e}^{-\left(r_{2} / \lambda_{m}\right)^{m}}
$$

where, $\lambda_{m}$ is the new length scale and $m(z)$ is a function of $z$-location:

$$
m(z)=2-z^{+} / R e_{\tau} .
$$

Note that close to the wall, $m \approx 2$ and the model (29) represents a Gaussian, whereas in the outer part of the boundary layer, $m \approx 1$, and the model is a simple exponential. 

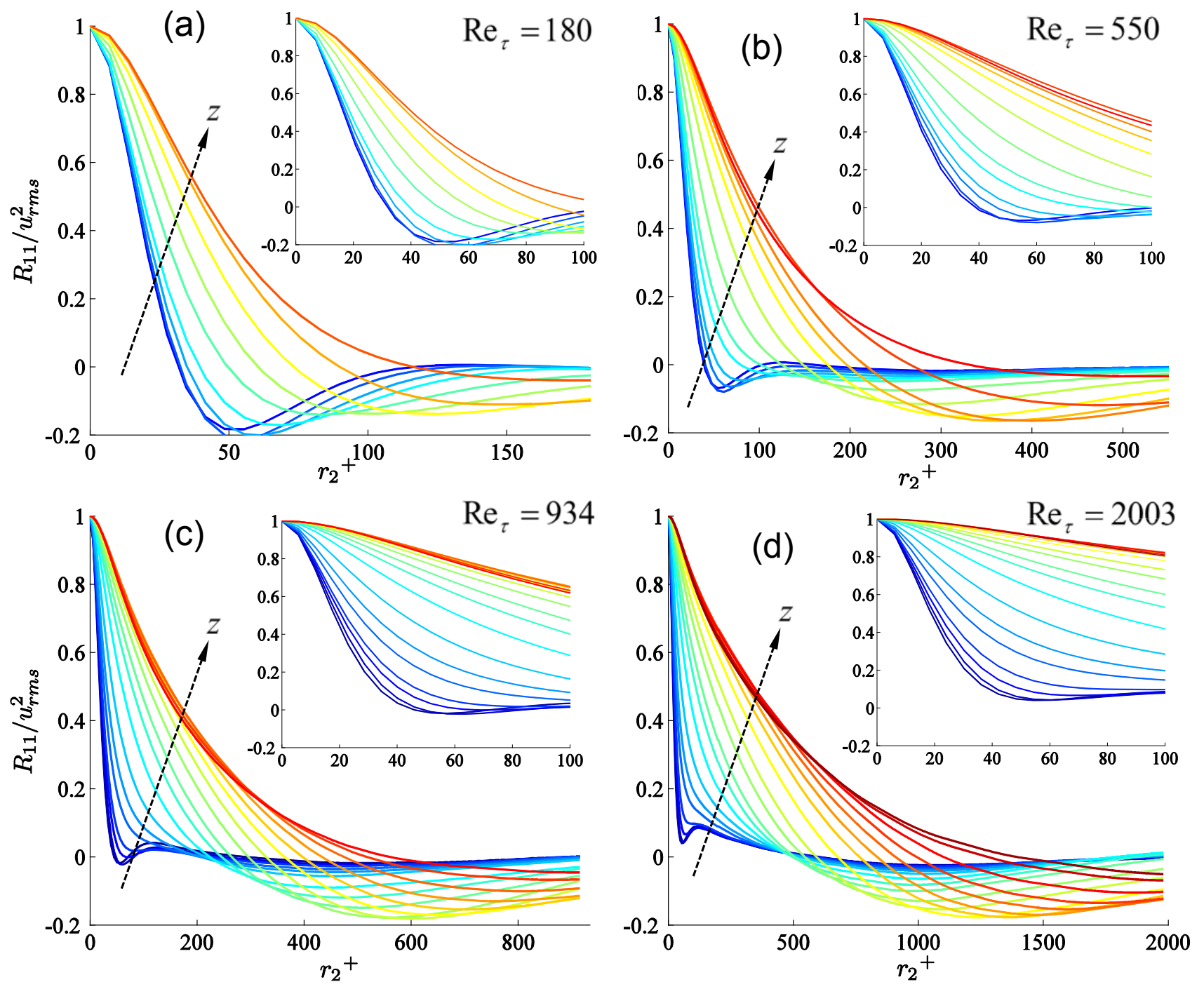

Figure 19. The normalized correlation, $R_{11} / u_{r m s}^{2}$ along spanwise direction, $r_{2}^{+}$; plotted up to $r_{2}^{+}=R e_{\tau}$. The inset is the expanded view plotted up to $r_{2}^{+}=100$. (a) $R e_{\tau}=180$, with $z^{+}=5,10,17,25,42,63,93,132$, and 186. (b) $R e_{\tau}=550$, with $z^{+}=5,9,15,20,30,39,61,104,165,221,277,414$ and 547. (c) $R e_{\tau}=934$, with $z^{+}$ as shown for figure 15. (d) $R e_{\tau}=2003$, with $z^{+}=6,10,14,20,31,40,60,100,151$, 200, 299, 403, 600, 804, 1002, 1204, 1401, 1606 and 1799.

The length scale $\lambda_{m}$ is defined in viscous scaling close to the wall, and in outer scaling away from the wall, and connected by a linear function in between:

$$
\lambda_{m}^{+}=\left\{\begin{array}{cl}
26 & \text { if } z^{+}<30 \\
\left(z^{+}-0.5 R e_{\tau}\right) & \left(26-0.2 R e_{\tau}\right) /\left(30-0.5 R e_{\tau}\right)+0.2 R e_{\tau} \\
& \text { if } 30 \leq z^{+}, z / \delta \leq 0.5 \\
0.2 R e_{\tau} \quad & \text { if } z / \delta>0.5 .
\end{array}\right.
$$

Substitution of (29) for a given $R e_{\tau}$ and $z$-location into (14) leads to,

$$
\frac{\tilde{u}_{r m s}^{2}}{u_{r m s}^{2}}=\frac{2}{l^{2}} \int_{0}^{l} \mathrm{e}^{-\left(s / \lambda_{m}\right)^{m}} \cdot(l-s) \mathrm{d} s,
$$



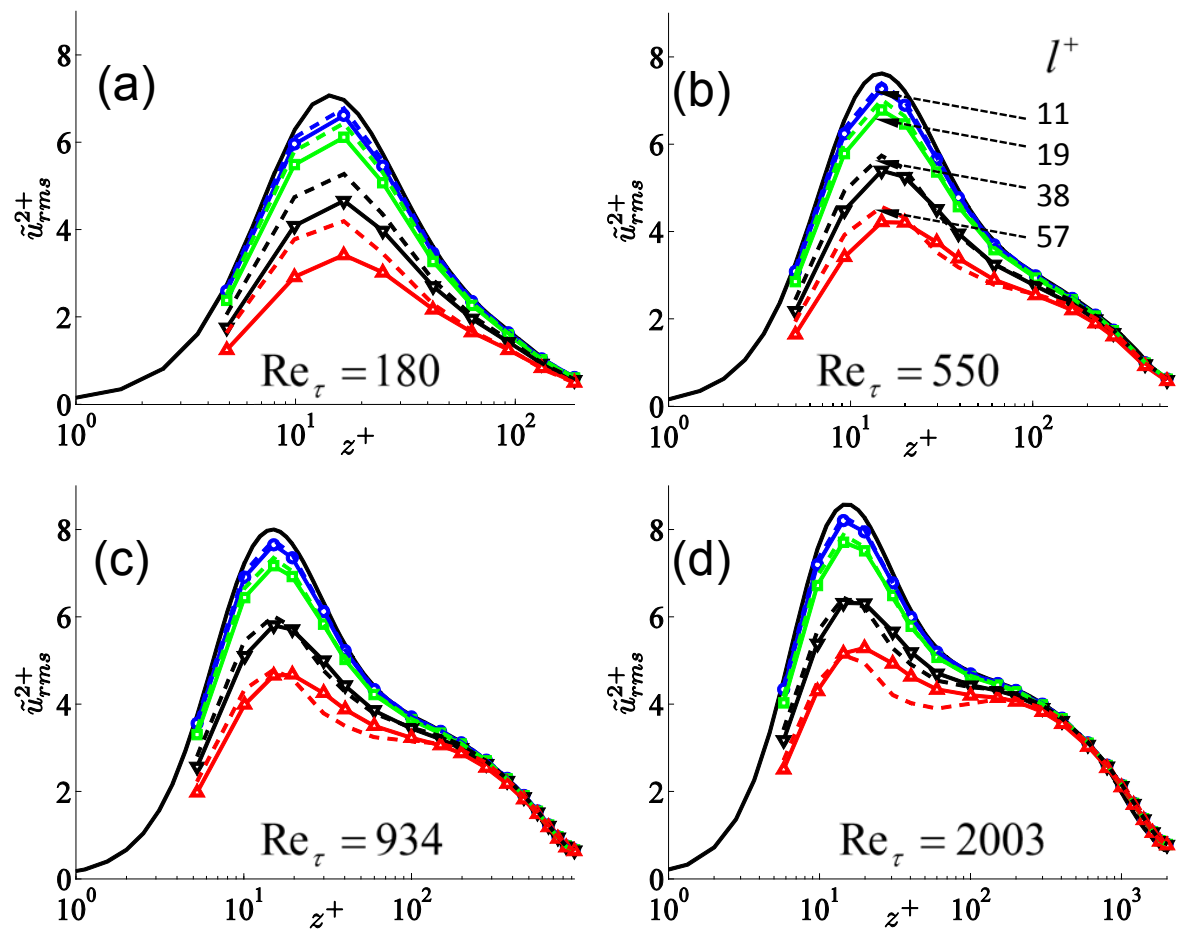

Figure 20. Comparison of spatial averaging obtained from DNS and model (33). (a) $R e_{\tau}=180$. (b) $R e_{\tau}=550$. (c) $R e_{\tau}=934$. (d) $R e_{\tau}=2003$.

where the integral can be evaluated by substituting $\left(s / \lambda_{m}\right)^{m}$ by another variable say, $t$. Note that both $m$ and $\lambda_{m}$ are positive quantities with $1 \leq m \leq 2$. The resulting form is,

$$
\frac{\tilde{u}_{r m s}^{2}}{u_{r m s}^{2}}=\frac{2 \lambda_{m}}{l m}\left[\gamma\left(\frac{1}{m},\left(\frac{l}{\lambda_{m}}\right)^{m}\right)-\left(\frac{\lambda_{m}}{l}\right) \gamma\left(\frac{2}{m},\left(\frac{l}{\lambda_{m}}\right)^{m}\right)\right],
$$

where, $\gamma(a, x)=\int_{0}^{x} \mathrm{e}^{-t} t^{a-1} \mathrm{~d} t$, is the incomplete Gamma function [e.g., Abramowitz and Stegun, 1964, p. 260]). Note that at the two extremes of $m$ the above expression simplifies. For $m=2$, the above equation reduces to (19), whereas the right hand side of $(33)$ becomes $\left(2 \lambda_{m} / l\right)\left[1-\left(\lambda_{m} / l\right)+\left(\lambda_{m} / l\right) \exp \left(-l / \lambda_{m}\right)\right]$ for $m=1$.

The attenuation estimated by the model (29) via (33) is compared with that obtained from DNS in figure 20(a)-(d) corresponding to $R e_{\tau}=180,550,934$ and 2003, respectively. The solid (black) line is the un-averaged distribution (at the DNS resolution), symbols are from DNS filtering and the dashed lines represent the model (29) along with (30) and (31). The predictions for all the higher Re is in general better than for the lowest one. This is due to the incomplete similarity of structures due to very low Re (as noted from figure 24 in Appendix B). Even with the crudeness of the model the overall shape and the peaks are not far from those obtained from DNS. Furthermore, the model works well for $l^{+}=19$, which is close to the usual hot-wire lengths that are commonly employed in experiments. 


\subsection{Comparison with some experimental data}

Thus far, all the analysis were concerned with averaged fields from numerical simulations or using theoretical models using a linear box-type filter. Though linear box-filters are commonly employed in studies of spatial resolution, it is not immediately obvious these accurately model the filtering of hot-wires in real flows. Here we are able to compare boxtype filtering of DNS with experimental data obtained with hot-wires at similar $l^{+}$and $R e_{\tau}$. Figures 21(a) and (b) present a one-to-one comparison of $\tilde{u}_{r m s}^{2+}$ from experiments in a channel flow with the DNS for different $l^{+}$and approximately the same $R e_{\tau}$ of 1000 and 2000, respectively. The filtered DNS data is shown with empty circles and experiments with filled symbols. The experimental data is taken from Ng et al. [2011], except for the highest $l^{+}$for both $R e_{\tau}$, which are newly conducted in the same channel facility as Ng et al. [2011]. Note that the DNS filtering data is somewhat sparse because the averaging is carried out at only those $z$-locations where the spectra are available. No comparison with the model is made here since it is already compared with the DNS simulations in figure 20. It can be observed that for low $l^{+}$the filtered DNS data match very well with that obtained from the experiments. However for larger $l^{+}$, say greater than $\approx 40$, small differences emerge between the DNS data and experiments, which become more prominent at $R e_{\tau}=2000$, even though the general trend seems to match for all the data presented. So, it can be safely concluded that for the hot-wires of length, $l^{+} \approx 20$, the comparison with linearly filtered signals is quite good, whereas for $l^{+} \gtrsim 40$ even the though overall trend is captured, discrepancies begin to appear.

Now that the efficacy of linear filtering is confirmed by the experiments, at least for $l^{+}$less than $\approx 40$, it is worth comparing some high $R e_{\tau}$ data with the model based on the assumptions of linear filtering. Figure 22 presents the peak variance of the steamwise velocity, $\left(\left.\tilde{u}_{r \operatorname{sm}}^{2+}\right|_{\max }\right)$ occurring at $z^{+} \approx 15$ from the model (33) based on the linear box-filtering and the correlation model (29) (broken lines), with various experimental data (symbols), for different $R e_{\tau}$ and at various $l^{+}$. The comparison can be considered fair considering the scatter in the experimental data involved and the degree of approximations that has gone into the correlation model (29). This implies that given the characteristic correlation scale, $\lambda^{+}=26$, close to the wall, the actual variances can be back-calculated from the measured variances.

\section{Summary and conclusions}

Single hot-wires are investigated here under the broader scope of spatial averaging of velocity measurements due to finite dimensionality of sensors in wall-bounded turbulence. A general framework for the study of spatial averaging of sensors is presented, specifically for hot-wires (single and multi-wires) and PIV planes which are classified depending upon their location, either in the homogeneous $(x y)$ plane, with no mean shear or in the inhomogeneous $(x z)$ planes. This part of the investigation delves into a detailed study of single hot-wires placed in the simplest case of homogeneous 

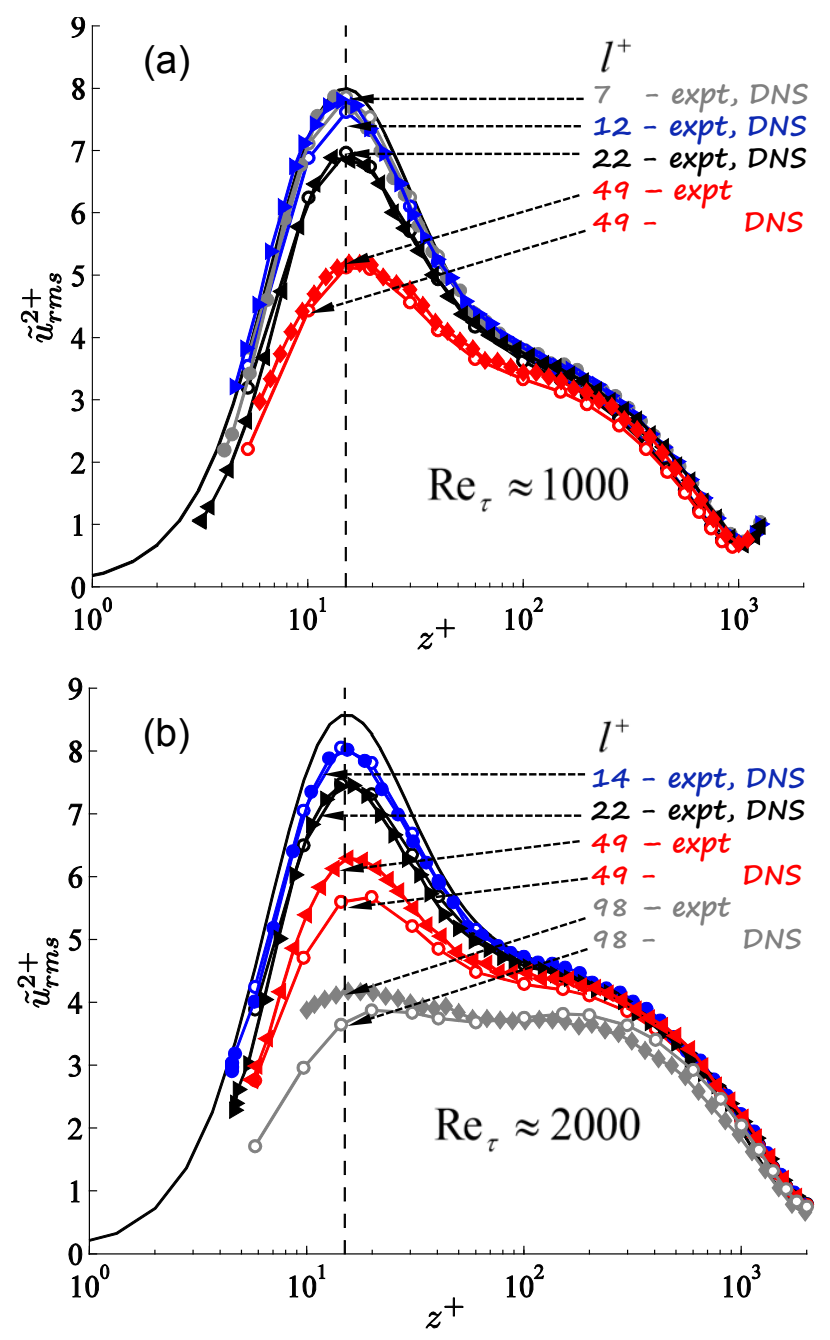

Figure 21. Comparison of averaged $\tilde{u}_{r m s}^{2+}$ obtained from the experiments and that from the DNS using box filtering, (a) at $R e_{\tau} \approx 1000$, and (b) at $R e_{\tau} \approx 2000$. Note that at $R e_{\tau} \approx 1000$ the experimental data for $l^{+}=7,12$ and 22 , and at $R e_{\tau} \approx 2000$ for $l^{+}=14,22$ and 49 are from $\mathrm{Ng}$ et al. [2011], whereas the highest $l^{+}$data for both the $R e_{\tau}$ are newly obtained. The dashed lines in both the figures are drawn at $z^{+}=15$.

plane, employing theoretical, numerical as well as experimental tools, with the view of extending the present work to other sensors in the homogeneous as well as inhomogeneous planes.

Spatial averaging is considered to be a linear filtering process with a generalized filter, and the averaged or filtered velocity field is represented as a convolution between the filter and the actual velocity field. It is shown that the averaged mean and fluctuating velocity components depend only on the corresponding un-averaged ones, with implications for $\times$-wires (such results are presented in Philip et al. [2013]). A general expression for the filtered two point correlation in terms of unfiltered correlation and the filter function is derived (in eqn (8)), and a corresponding one for the spectrum (eqn (10)). Thereafter, a simplified filter of the form of a 'box-filter' is chosen (which approximates the actual hot-wires quite well as is confirmed later by experimental data) 


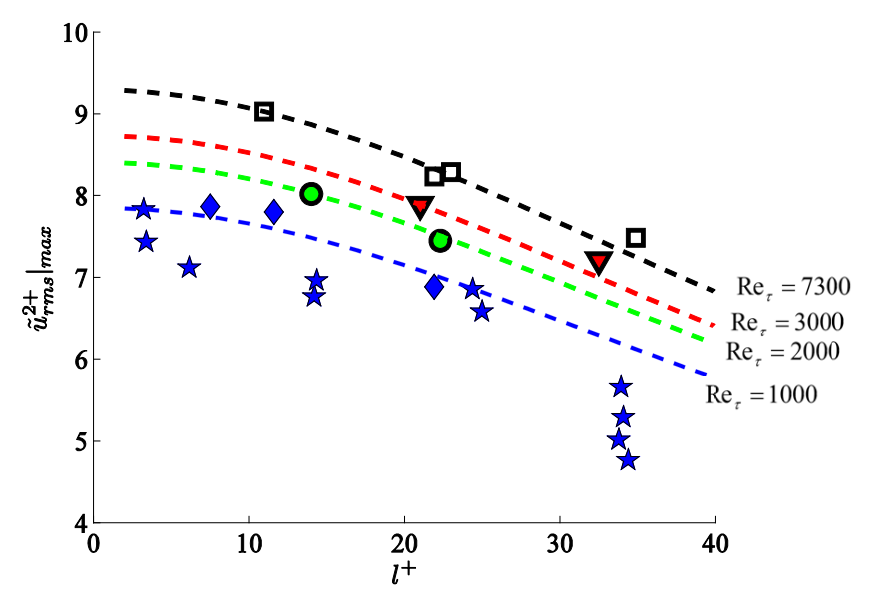

Figure 22. Comparison of model (33), in lines, with the experimental distribution of the near-wall peak $\left(\left.\tilde{u}_{r m s}^{2+}\right|_{\max }\right)$, in symbols, for varying $l^{+}$at selected Reynolds numbers. The symbols, $\star$, for $R e_{\tau} \approx 1000$, from Ligrani and Bradshaw [1987] and from $\mathrm{Ng}$ et al. [2011]; $\circ$ for $R e_{\tau} \approx 2000$, from $\mathrm{Ng}$ et al. [2011]; the symbols, $\boldsymbol{\nabla}$, for $R e_{\tau} \approx 3000$, from $\mathrm{Ng}$ et al. [2011], and $\square$ for $R e_{\tau} \approx 7300$, from Nickels et al. [2007] and Hutchins et al. [2009].

allowing us to proceed with more concrete expressions for the averaged correlation, spectrum and $u_{r m s}^{2}$. Some of these expressions are already known, however, here they are presented in a unified manner starting from a arbitrary linear filter. These expressions are validated using the DNS channel flow database of del Álamo and Jiménez [2003] at $R e_{\tau}=934$ (figures 7 (a), (b) and (c)).

Since the two-point correlation along the wire-length over its distance $l$ dictates the velocity attenuation, a simple model of correlation is proposed over the length $l$, characterized by the correlation length scale, $\lambda$. This model has been used to derive explicit relations for the averaged correlation, spectrum and $u_{r m s}^{2}$ in terms of the un-averaged ones (equations 17, 18 and 19), and these relations compare well with the results obtained from the averaging of DNS data, clarifying to some extent the empiricism involved in the work of Chin et al. [2009]. Furthermore, we have also shown why the 'missing spectra' takes the approximate form of a Gaussian as modelled by Chin et al. [2009]. The model exemplifies the fact that the appropriate length scale for investigating the spatial resolution in hot-wires is the characteristic correlation length scale $\lambda$, rather than the Taylor micro-scale or the viscous length scales. However, since $\lambda$ scales with the viscous units close to the wall (and outer scaling away from the wall), there is no difference for spatial resolution studies using $\lambda$, or $\nu / u_{\tau}$ close to the wall; this is of course not true away from the wall where $\lambda$ is the only representative length scale. The model of the correlation is extended for different $z$-locations and $R e$, with the cental purpose of understanding the averaging effects rather than as a technique for 'predicting' the un-averaged statistics from the averaged ones. The model agrees reasonably well with the DNS calculations. Since the analysis is based on the correlations, the expression 

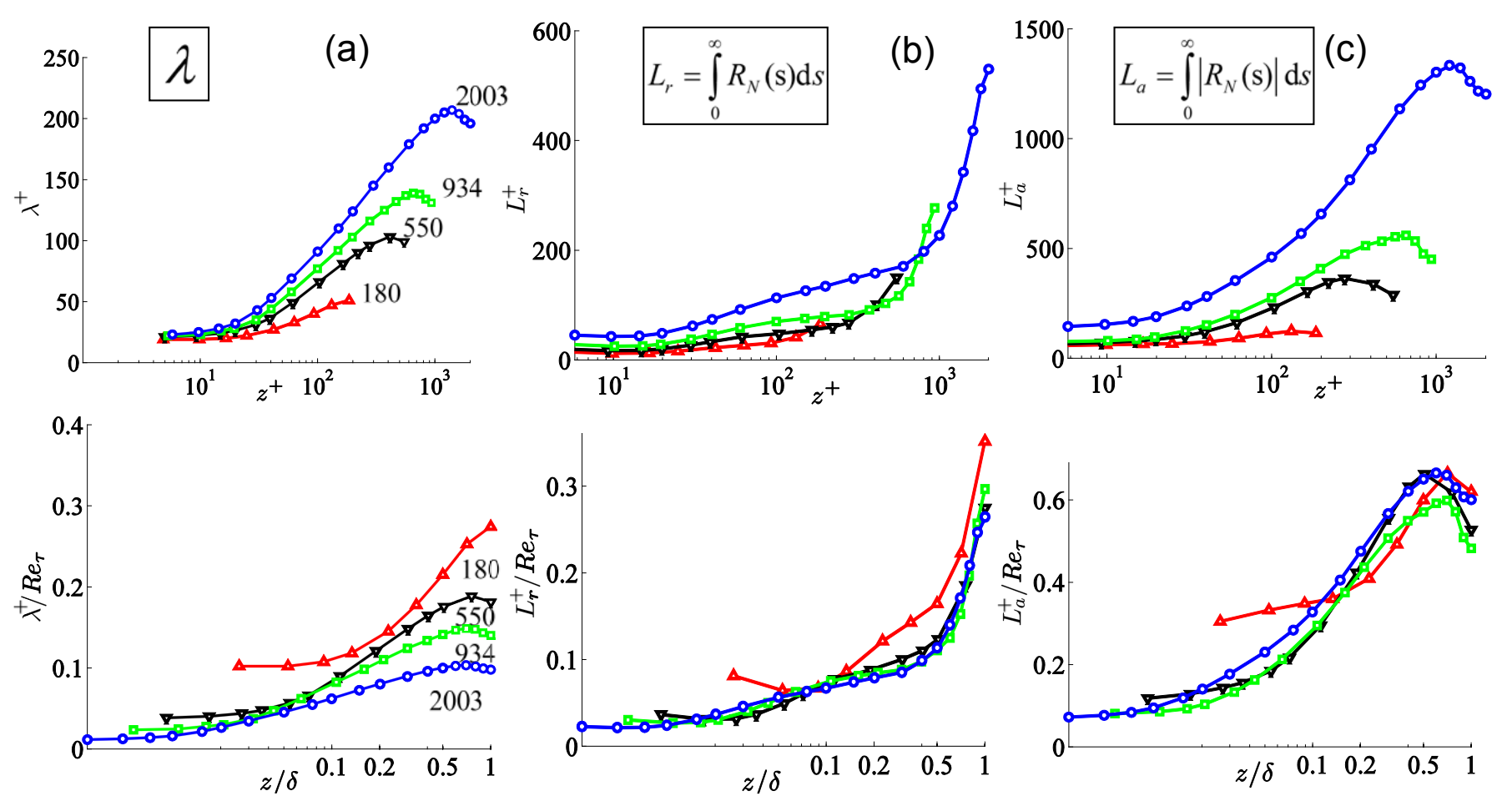

Figure 23. Different length scales in inner scaling - top panel and outer scaling bottom panel. (a) Distribution of $\lambda$, (b) $L_{r}$ and (c) $L_{a}$.

derived here is equally valid for other flows too, laminar or turbulent, and not restricted to wall-turbulence (the only flow considered in the present work).

Finally, the efficacy of linear filtering is determined by comparing the experimental data taken in a channel flow at $R e_{\tau}$ of 1000 and 2000 with the linearly filtered data from DNS for different $l^{+}$. There is sufficient evidence that for $l^{+} \lesssim 40$, the linear filtering reproduces the experimental data very well; however, with increasing $l^{+}$and $R e$, small differences develop. Despite these, there is still an approximate correspondence between experiments and linearly filtered data for even higher $l^{+}$(figure 21). Furthermore, the approximate correlation model with the characteristic length scale, $\lambda$, developed for low $R e$ seems to estimate the maximum in $u_{r m s}^{2+}$ quite well for experimental data from various sources at relatively high Reynolds numbers, confirming the suitability of $\lambda$ as the appropriate length scale for spatial averaging in hot-wires.

\section{Acknowledgments}

The authors gratefully acknowledge support from the Australian Research Council. 


\section{Appendix A: Some definitions and the expression for $\tilde{R}_{i j}(r)$}

Defining Fourier transform of a function $\mathcal{F}[g(x)]=: \hat{g}(k)$ and its inverse $g(x)$ respectively, as [e.g., Pope, 2000],

$$
\begin{aligned}
& \hat{g}(k)=\frac{1}{2 \pi} \int_{-\infty}^{\infty} g(x) \mathrm{e}^{-i k x} \mathrm{~d} x, \quad \text { and }, \\
& g(x)=\int_{-\infty}^{\infty} \hat{g}(x) \mathrm{e}^{i k x} \mathrm{~d} k,
\end{aligned}
$$

where, $k$ is the wavenumber, the convolution and correlation theorems take the form (with $f$ as another function),

$$
\mathcal{F}[g * f]=2 \pi \hat{g} \hat{f}
$$

and,

$$
\mathcal{F}[g \star f]=2 \pi \hat{g}^{*} \hat{f}
$$

where, the symbol $\star$ is for correlation and the superscript * represents complex conjugate. Substituting the expression for the averaged field from eqn (5) into eqn (7), one obtains [also see Bracewell, 2000, chapter 17],

$$
\begin{aligned}
\tilde{R}_{i j}(r) & =\frac{1}{l^{2}} \lim _{L \rightarrow \infty} \frac{1}{L}\left(u_{i}^{\prime} * b_{I}\right) \star\left(u_{j}^{\prime} * b_{J}\right) \\
& =\frac{1}{l^{2}} \lim _{L \rightarrow \infty} \frac{1}{L}\left(u_{i}^{\prime} * b_{I}\right)_{r} *\left(u_{j}^{\prime} * b_{J}\right) \\
& =\frac{1}{l^{2}} \lim _{L \rightarrow \infty} \frac{1}{L}\left(u_{i r}^{\prime} * b_{I r} * u_{j}^{\prime} * b_{J}\right) \\
& =\frac{1}{l^{2}} \lim _{L \rightarrow \infty} \frac{1}{L}\left(u_{i r}^{\prime} * u_{j}^{\prime}\right) *\left(b_{I r} * b_{J}\right) \\
& =\frac{1}{l^{2}} \lim _{L \rightarrow \infty} \frac{1}{L}\left(u_{i}^{\prime} \star u_{j}^{\prime}\right) *\left(b_{I} \star b_{J}\right) \\
& =\frac{1}{l^{2}} R_{i j} *\left(b_{I} \star b_{J}\right)
\end{aligned}
$$

where, $g_{r}(x)$ is the short notation for the reverse of $g(x), g_{r}(x):=g(-x)$. In the above derivation, the property that, $f \star g=f_{r} * g$ and the associative property of convolutions, $(f * g) * h=f *(g * h)$ have been employed.

\section{Appendix B: A model for the spanwise two-point correlation parameterised for varying $z$ and $R e$}

Here we present an example of a model for the spanwise two-point correlation, extending the initial model given by eqn (15) such that it is a function of $z$ and $R e$.

The principle quantity that is of interest in modelling the spatial averaging using the model (15) is $\lambda$. Figure 23(a) presents the distribution of $\lambda^{+}$with inner scaling (along $z^{+}$) in the top panel whereas the bottom one shows the same data in outer scaling, i.e., $\lambda^{+} / R e_{\tau}$ against $z / \delta$. As expected the values of $\lambda$ for all $R e$ collapse in the inner scale for small $z^{+}$, however, there does not seem to be a convincing collapse in 
outer scaling. From the discussion of correlations we would have expected $\lambda$ to scale with outer scaling for large $z / \delta$ close to the centerline. This casts doubt on the way the length scale $\lambda$ is defined, which as already mentioned is by fitting the model (15) to the correlations up to $r_{2}^{+}=100$. For comparison and to contrast, figures 23(b) and (c) show the regular integral length scale $\left(L_{r}\right)$ as well as the integral scale based on the absolute value of the correlation $\left(L_{a}\right)$, both defined respectively as $L_{r}^{+}:=\int_{0}^{\infty} R_{N}\left(r_{2}^{+}\right) \mathrm{d} r_{2}^{+}$and $L_{a}^{+}:=\int_{0}^{\infty}\left|R_{N}\left(r_{2}^{+}\right)\right| \mathrm{d} r_{2}^{+}$. Note that the upper integration limit, $\infty$, in reality is limited by the DNS domain size. The top and the bottom panels are the same data plotted in inner and outer scaling respectively. Both $L_{r}$ and $L_{a}$ do not scale with plus units at any $z$-location. This is expected because the 'integral' scale employs limits of integration which extend to very large scales. However, $L_{a}$ at least shows the general shape which is similar to $\lambda$ in inner scaling. In the outer scaling both $L_{r}$ and $L_{a}$ show a good collapse throughout the $z$-locations and $L_{a}$ especially in the outer region, with the slight exception of the lowest $R e$.

Physically, the variation of the fluctuating velocity along the hot-wire (in the spanwise direction) is the cause for attenuation. Close to the wall the fluctuating velocity patterns are caused by the streaky structures. Further away from the surface these are due to the larger scale bulges in the outer layer. None of the length scales, $L_{r}, L_{a}$ or $\lambda$ capture this phenomenon completely. Though $\lambda$ does capture the near-wall streaks it fails in the outer region. A second look at the correlations in figure 19 is helpful in deciphering the deficiencies in the definition of $\lambda$, or the model in (15). Two problems present themselves. First, even though a Gaussian type model (15) is appropriate for near-wall $z$-locations, it fails to capture the distribution in the outer region (c.f. figure 19) which is not of Gaussian type. In fact, a Gaussian model fits close to the wall region because the near-wall streaks reside there. There are no such 'dominant' structures present in the outer region and the correlation is composed of many such uncorrelated structures of varying scales. A correlation of the type of decaying exponential represents such a process well, and this is also observed in figure 19, where for large $z$-locations the correlations indeed fall off like $\mathrm{e}^{-\left(r / \lambda_{1}\right)}$. In fact, a decaying exponential is the common model used for correlation in turbulence. In short, the correlation close to the wall is of the type, $\mathrm{e}^{-(r / \lambda)^{2}}$, whereas close to $z / \delta$ it is of the form $\mathrm{e}^{-(r / \lambda)}$, which encourages one to model the correlation as,

$$
R_{N}\left(r_{2}\right)=\mathrm{e}^{-\left(r_{2} / \lambda_{m}\right)^{m}}
$$

where, $\lambda_{m}$ is the new length scale and $m(z)$ is a function of $z$-location, with $m=2$ close to the wall and becoming $m=1$ at the centreline. The simplest function to achieve this is a linear variation, and to this end, $m$ can be written as,

$$
m(z)=2-z^{+} / R e_{\tau}
$$

This addresses the first problem. Secondly, note that figure 19 is plotted for $r_{2}^{+}$up to $R e_{\tau}$, from which we note similar correlation curves for different $R e$ at large $z$-locations. In modelling (15), we restricted the fit up to $r_{2}^{+}=100$; this needs to be relaxed for correlations close to the centreline. Therefore, the range of correlation in which the 

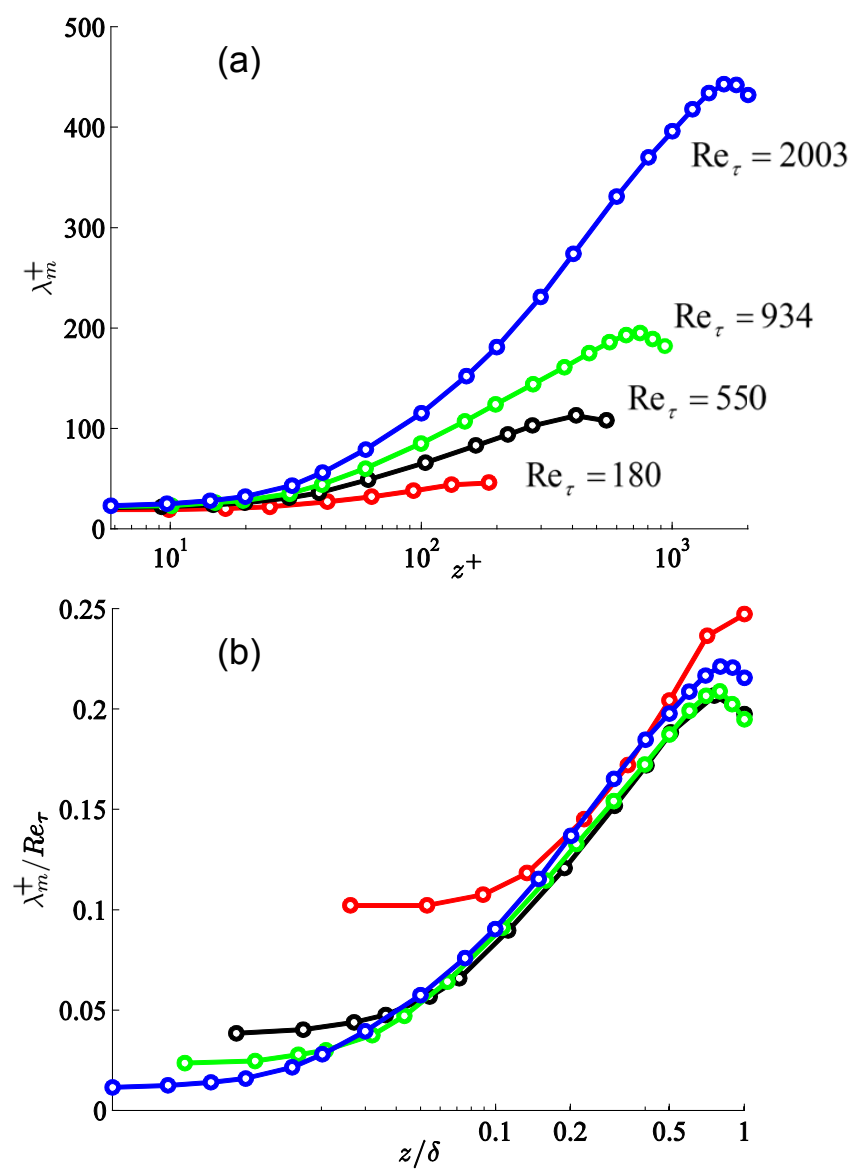

Figure 24. Distribution of $\lambda_{m}$ in (a) inner scaling (b) outer scaling.

model $(29)$ needs to be fitted must vary from about $r_{2}^{+} \approx 100$ close to the wall to $r_{2}^{+} \approx R e_{\tau}$ at the centreline, and again, a linear variation is sought such that the range of fitting the model (29) follows the function, $\left(z^{+} / R e_{\tau}\right)\left(R e_{\tau}-100\right)+100$.

With the above considerations of $m(z)$ and the range, figures 24(a) and (b) show the variation of $\lambda_{m}$ with $z$ in inner and outer scaling, respectively. As observed for $\lambda$ before, $\lambda_{m}$ does not change with $R e$ close to the wall for inner scaling, however, unlike $\lambda$, $\lambda_{m}$ does not change with $R e$ far from the wall. Thus the scaling of $\lambda_{m}$ follows the usual viscous scaling close to the wall and outer scaling away from it. This also implies that we can model $\lambda_{m}$ with the usual inner-outer scaling. One such proposal is presented in figure 25, which shows the data from figure 24 plotted in inner scaling for $z^{+}<30$ and the rest of the data in outer scaling. It can be inferred that for $z^{+}<30, \lambda_{m}^{+}$ takes on approximately a constant value, say $\approx 26$, whereas for $z / \delta>0.5, \lambda_{m}^{+} / R e_{\tau}$ (i.e. in outer scaling) is again approximately constant, say $\approx 0.2$. The in-between region, $30 \geq z^{+}, z / \delta \leq 0.5$, can, as a first approximation be represented by linearly connecting the two extreme regions. In fact the linear variation of spanwise lengthscales of conditional eddies was found by Tomkins and Adrian [2000]. Compactly, the 


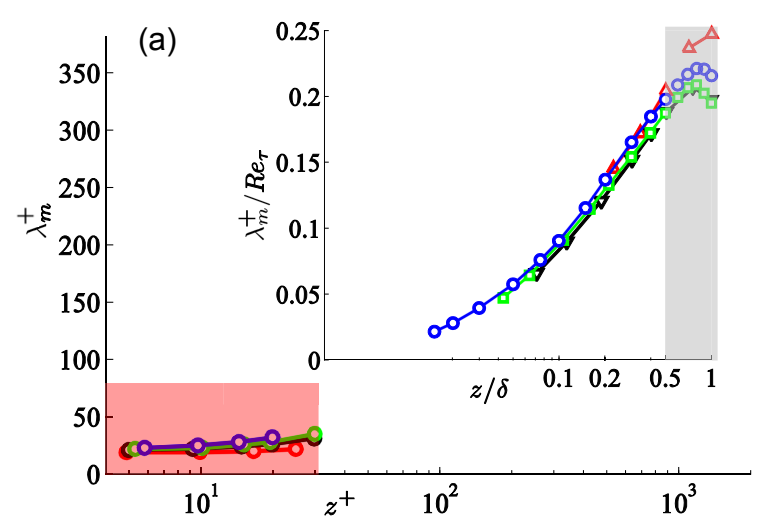

(b)

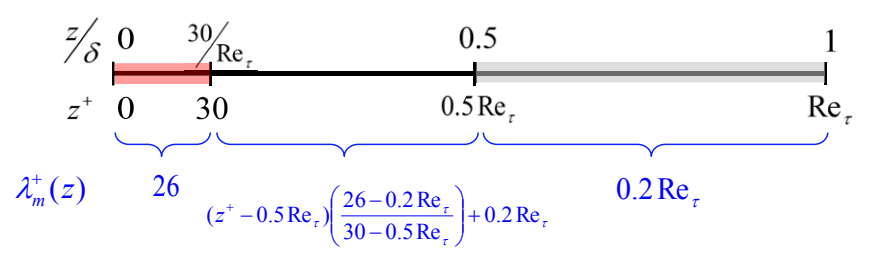

Figure 25. A model for $\lambda_{m}$. (a) Distribution of the same data as in figure 24 with $\lambda_{m}$ in inner scaling for $z^{+}<30$ and the inset has $\lambda_{m}$ in outer scaling for the rest of the data. (b) Schematic of the model for $\lambda_{m}$ as a function of $z$.

distribution of $\lambda_{m}^{+}$can be written as,

$$
\lambda_{m}^{+}=\left\{\begin{array}{cl}
26 & \text { if } z^{+}<30 \\
\left(z^{+}-0.5 R e_{\tau}\right) & \left(26-0.2 R e_{\tau}\right) /\left(30-0.5 R e_{\tau}\right)+0.2 R e_{\tau} \\
& \text { if } 30 \leq z^{+}, z / \delta \leq 0.5 \\
0.2 R e_{\tau} \quad & \text { if } z / \delta>0.5 .
\end{array}\right.
$$

This is also shown schematically in figure 25(b). Thus, eqn (29) along with (30) and (31) furnishes a first model for correlation for any given Reynolds number (at least in turbulent channel flows). Note that the Taylor microscale $\lambda_{T}$ also scales in inner scaling close to the wall and in outer scaling away from the wall [e.g. Segalini et al., 2011b] similar to $\lambda_{m}$. Furthermore, Segalini et al. [2011b] have shown that $\lambda_{T}$ seem to scale with $\sqrt{R e_{\tau}}$ and a similar possibility might exist for $\lambda_{m}$.

\section{References}

M. Abramowitz and I. A. Stegun. Handbook of Mathematical Functions with Formulas, Graphs, and Mathematical Tables. Dover, New York, ninth dover printing, tenth gpo printing edition, 1964.

R. J. Adrian and J. Westerweel. Particle Image Velocimetry. Cambridge University Press, 2011.

S. C. C. Bailey, G.G.Kunkel, M. Hultmark, M. Vallikivi, J. P. Hill, K. A. Meyer, C. Tsay, C. B. Arnold, and A. J. Smits. Turbulence measurements using a nanoscale thermal anemometry probe. J. Fluid. Mech, 663:160-179, 2010. 
R. N. Bracewell. The Fourier transform and its applications. McGraw-Hill Companies, Inc., 2000.

H. H. Bruun. Hot-wire anemometry. Principles and Signal Analysis. Oxford University Press, 1995.

J. D. Cameron, S. C. Morris, S. Bailey, and A. J. Smits. Effects of hot-wire length on the measurement of turbulent spectra in anisotropic flows. Meas. Sci. Technol., 21: 105407, 2010.

J Carlier and M Stanislas. Experimental study of eddy structures in a turbulent boundary layer using particle image velocimetry. J. Fluid. Mech, 535:143-188, 2005.

C. C. Chin, N. Hutchins, A. S. H. Ooi, and I. Marusic. Use of direct numerical simulation (DNS) data to investigate spatial resolution issues in measurements of wall-bounded turbulence. Meas. Sci. Technol., 20:115401, 2009.

C. C. Chin, N. Hutchins, A. S. H. Ooi, and I. Marusic. Spatial resolution correction for hot-wire anemometry in wall turbulence. Exp. Fluids, 50:1443-1453, 2011.

G. Comte-Bellot and J. F. Foss. Thermal Anemometry, chapter 5.2, pages 229-286. Springer, Springer-Verlag Berlin Heidelberg, 2007.

S. Corrsin and L. S. G. Kovasznay. On the hot-wire length correction. Phys. Rev., 75: 1954, 1949.

J. C. del Álamo and J. Jiménez. Spectra of the very large anisotropic scales in turbulent channels. Phys. Fluids, 15:L41-L44, 2003.

J. C. del Álamo, J. Jiménez, P. Zandonade, and R. D. Moser. Scaling of the energy spectra of turbulent channels. J. Fluid. Mech, 490:37-74, 2003.

H. L. Dryden, G. B. Schubauer, Jr. C. Mock, and H. K. Skramstad. Measurements of intensity and scale of wind tunnel turbulence and their relation to the critical reynolds number of spheres. Technical report, NACA Report 581, 1937.

F. Frenkiel. The influence of the length of a hot wire on the measurements of turbulence. Phys. Rev, 75:1263-1264, 1949.

F. Frenkiel. Effects of wire length in turbulence investigations with a hot-wire anemometer. Aero. Quart., 5:1-24, 1954.

M. Hultmark, A. Ashok, and A. J. Smits. A new criterion for end-conduction effects in hot-wire anemometry. Meas. Sci. Technol., 22:055401, 2011.

N. Hutchins, T. B. Nickels, I. Marusic, and M. S. Chong. Hot-wire spatial resolution issues in wall-bounded turbulence. J. Fluid. Mech, 635:103-136, 2009.

A. V. Johansson and P. H. Alfredsson. Effects of imperfect spatial resolution on measurements of wall-bounded turbulent shear flows. J. Fluid. Mech, 137:409-421, 1983.

P. Lavoie, G. Avallone, F. De Gregorio, G. P. Romano, and R. A. Antonia. Spatial resolution of piv for the measurement of turbulence. Exp. Fluids, 43:39-51, 2007. 
P. M. Ligrani and P. Bradshaw. Spatial resolution and measurement of turbulence in the viscous sublayer using subminiature hot-wire probes. Exp. Fluids, 5:407-417, 1987.

I. Marusic and R. A. Adrian. Eddies and scales of wall turbulence. Cambridge University Press, Cambridge, 2013.

P. Moin and P. R. Spalart. Contributions of numerical simulation data bases to the physics, modeling, and measurement of turbulence. Technical report, NASA Tech. Memo 100022, 1987.

P. A. Monkewitz, R. D. Duncan, and H. M. Nagib. Correcting hot-wire measurements of stream-wise turbulence intensity in boundary layers. Phys. Fluids, 22:091701, 2010.

H. C. H. Ng, J. P. Monty, N. Hutchins, M. S. Chong, and I. Marusic. Comparison of turbulent channel and pipe flows with varying Reynolds number. Exp. Fluids, 51: 1261-1281, 2011.

T. B. Nickels, I. Marusic, S. Hafez, N. Hutchins, and M. S. Chong. Some predictions of the attached eddy model for a high Reynolds number boundary layer. Phil. Trans. R. Soc. A, 365:807-822, 2007.

A. E. Perry. Hot-wire anemometry. Oxford University Press, 1982.

J. Philip, R. Baidya, N. Hutchins, J. P. Monty, and I. Marusic. Spatial averaging of streamwise and spanwise velocity measurements in wall-bounded turbulence using $\vee$ and $\times$-probes. Meas. Sci. Technol., in press, 2013.

S. B. Pope. Turbulent flows. Cambridge University Press, 2000.

N. Saikrishnan, I. Marusic, and E. K. Longmire. Assessment of dual plane piv measurements in wall turbulence using DNS data. Exp. Fluids, 41:265-278, 2006.

A. Segalini, A. Cimarelli, J-D. Rüedi, E. De Angelis, and A. Talamelli. Effect of the spatial filtering and alignment error of hot-wire probes in a wall-bounded turbulent flow. Meas. Sci. Technol., 22:105408, 2011a.

A. Segalini, R. Örlü, P. Schlatter, P. H. Alfredsson, J-D Rüedi, and A. Talamelli. A method to estimate turbulence intensity and transverse taylor microscale in turbulent flows from spatially averaged hot-wire data. Exp. Fluids, 51:693-700, 2011b.

A. J. Smits, J. Monty, M. Hultmark, S. C. C. Bailey, N. Hutchins, and I. Marusic. Spatial resolution correction for wall-bounded turbulence measurements. J. Fluid. Mech, 676:41-53, 2011.

Y. Suzuki and N. Kasagi. Evaluation of hot-wire measurements in wall shear turbulence using a direct numerical simulation database. Exp. Therm. Fluid Sci., 5:69-77, 1992.

C. D. Tomkins and R. J. Adrian. Spanwise structure and scale growth in turbulent boundary layers. J. Fluid. Mech, 500:135-144, 2000.

M. S. Uberoi and L. S. G. Kovasznay. On mapping and measurement of random fields. Q. Appl. Math, 10:375-393, 1953.

J. C. Wyngaard. Measurement of small-scale turbulence structure with hot wires. J. Phys. E: Sci. Instrum., 1:1105-1108, 1968. 
V. Yakhot, S. C. C. Bailey, and A. J. Smits. Scaling of global properties of turbulence and skin friction in pipe and channel flows. J. Fluid. Mech, 652:65-73, 2010. 